\title{
A PRIORI ERROR ESTIMATES FOR THE SPACE-TIME FINITE ELEMENT APPROXIMATION OF A QUASILINEAR GRADIENT ENHANCED DAMAGE MODEL
}

\author{
Marita Holtmannspötter*
}

\begin{abstract}
In this paper we investigate a priori error estimates for the space-time Galerkin finite element discretization of a quasilinear gradient enhanced damage model. The model equations are of a special structure as the state equation consists of two quasilinear elliptic PDEs which have to be fulfilled at almost all times coupled with a nonsmooth, semilinear ODE that has to hold true in almost all points in space. The system is discretized by a constant discontinuous Galerkin method in time and usual conforming linear finite elements in space. Numerical experiments are added to illustrate the proven rates of convergence.
\end{abstract}

Mathematics Subject Classification. 65J08, 65M12, 65M15, 65M60.

Received May 6, 2020. Accepted April 26, 2021.

\section{INTRODUCTION}

In this paper, we derive a priori error estimates for the space-time finite element discretization of a quasilinear gradient enhanced damage model. To be more specific, we investigate the finite element approximation of the quasilinear damage model

$$
\begin{aligned}
-\operatorname{div}(g(\varphi(t)) \mathbb{C} \varepsilon(\boldsymbol{u}(t))) & =l(t) & & \text { in } \boldsymbol{W}^{-1, p}(\Omega) \\
-\alpha \Delta \varphi(t)+\beta \varphi(t)+\frac{1}{2} g^{\prime}(\varphi(t)) \mathbb{C} \varepsilon(\boldsymbol{u}(t)): \varepsilon(\boldsymbol{u}(t)) & =\beta d(t) & & \text { in } H^{1}(\Omega)^{*} \\
\partial_{t} d(t)-\frac{1}{\delta} \max \{-\beta(d(t)-\varphi(t))-r, 0\} & =0 & & \text { a. e. in } \Omega \\
d(0) & =d_{0} & &
\end{aligned}
$$

for almost all $t \in[0, T]$. A precise formulation and an explanation of the given data will be given in the next section. The model is motivated by a specific gradient enhanced damage model, first developed in $[5,6]$ and (after a slight modification that is more amenable) thoroughly analyzed for a mathematical point of view in $[25,26]$. It describes the displacement $u$ of a body $\Omega$ influenced by a given force $l$. The model features two damage variables $\varphi$ and $d$ where the first one is more regular in space whereas the second one carries the evolution of damage in

Keywords and phrases. Error estimates, finite elements, quasilinear coupled PDE-ODE system, damage material model.

Faculty of Mathematics, University of Duisburg-Essen, 45127 Essen, Germany.

*Corresponding author: marita.holtmannspoetter@uni-due.de

(C) The authors. Published by EDP Sciences, SMAI 2021

This is an Open Access article distributed under the terms of the Creative Commons Attribution License (https://creativecommons.org/licenses/by/4.0), which permits unrestricted use, distribution, and reproduction in any medium, provided the original work is properly cited. 
time. Both are coupled by a penalty term in the free energy functional with $\beta$ being the penalty parameter. The parameter $\alpha$ originates from the gradient enhancement while $\delta$ is a viscosity parameter (see [25] for details). The resulting system consists of two nonlinear PDEs which have to hold true in almost all time points and an ODE that should be fulfilled in almost every point in space. All three equations are fully coupled with one another. In [25], existence of a unique solution for a fixed load $l$ is proven under the additional assumption that the penalty parameter $\beta$ is sufficiently large.

This contribution focuses on the numerical analysis of the model equations. We will use constant discontinuous finite elements for the discretization in time and usual $H^{1}$-conforming linear finite elements for the discretization in space. For a first analysis of the discretization of such a model we simplified the underlying PDE system, skipping the displacement variable $u$ as well as the nonlinear material function, see [19] for details. A further simplified linear version of this model, that lacks the max-operator in the ODE, has been studied in a companion paper, see [20]. The aim of this paper is to establish a priori discretization error estimates for the finite element discretization of (1.1)-(1.4). We will rely on the results already established for the simplified versions of this damage model and point out the difficulties that arise if we try to adapt the strategies to the original damage model. The nonsmooth right-hand side of the ODE as well as the quasilinear coupling will be of special interest.

Let us put our work into perspective: On the one hand, starting with Griffith's model [16] for brittle fracture various models have been developed and improved for damage and crack propagation, see $[4,7,14,15,22]$ among others and the references therein. In contrast to our damage model these contributions all have in common that they feature models with only one damage variable. Both model types have been used for numerical simulations, see $[1,3,23,28,30]$ but to the best of the authors knowledge a numerical analysis regarding a priori error estimates for the discretization of such models has not been investigated so far. On the other hand, there are of course contributions about the numerical analysis of parabolic equations, see for example [9-13] for uncontrolled equations or $[24,29]$ in the context of optimal control. All of these works consider parabolic PDEs with a simpler structure than our damage model. The only contribution known to the author regarding numerical analysis of a damage model is the contribution [27], which considers a linearized, time-discrete phase-field model for crack propagation in the context of optimal control. Finally, we would like to mention [18], which (among other more general results) provides regularity results for time-discrete phase-field models that are applicable in our case as well and are a key ingredient for the spatial error estimation.

The paper is organized as follows: In section two we state the exact setting of the damage problem and briefly summarize the known results from the analysis of the continuous damage model. In section three, we focus on the semidiscrete (time-discrete) damage problem and prove linear convergence in time while in section four we establish a convergence rate of up to $\mathcal{O}\left(h^{\frac{3}{2}-\varepsilon}\right)$ in space of our discretization. The last section presents a numerical example.

\section{The COntinuous DAMAGE MODEL}

In this section we establish the principal assumptions on the data as well as the used notation and present basic analytical results.

Throughout this paper, let $\Omega \subset \mathbb{R}^{2}$, be a bounded convex polygonal domain with boundary $\partial \Omega=\Gamma_{D} \cup \Gamma_{N}$. $\Gamma_{D}$ and $\Gamma_{N}$ should be disjoint sets of positive measure with $\Gamma_{N}$ being relatively open while $\Gamma_{D}$ is supposed to be relatively closed. In addition $\Omega \cup \Gamma_{N}$ has to be regular in the sense of Gröger, see [17]. The time interval will be denoted by $I:=(0, T)$ where $T>0$ is a given real number.

Moreover, let $\alpha, \beta, \delta, r>0$ be given parameters. The last parameter $r$ denotes the fracture toughness of the material. As already mentioned in the introduction, the system is only uniquely solvable for $\beta$ sufficiently large, cf. [25], Assumption 3.16. Therefore, we also impose the following:

\section{Assumption 2.1. The parameter $\beta$ is sufficiently large with the threshold depending only on the given data.}

The size of $\beta$ depends - among other - on the norm of $l$, cf. [33], Remark 3.2. Therefore, to fulfill Assumption 2.1, we will often restrict the choice of $l$ to an open ball with fixed radius $\rho$. 
The function $g: \mathbb{R} \rightarrow[\epsilon, 1]$ should belong to $C^{2}(\mathbb{R})$ with $g^{\prime}, g^{\prime \prime} \in C^{0,1}(\mathbb{R}) \cap L^{\infty}(\mathbb{R})$ and $\epsilon>0$. Nemytskiioperators associated to $g$ will again be denoted by $g$. The fourth-order tensor $\mathbb{C} \in L^{\infty}\left(\Omega ; \mathcal{L}\left(\mathbb{R}_{\mathrm{sym}}^{2 \times 2}\right)\right)$ is assumed to be symmetric and uniformly coercive, i.e., there exists a constant $\gamma_{\mathbb{C}}>0$ such that

$$
\mathbb{C}(x) \boldsymbol{\sigma}: \boldsymbol{\sigma} \geq \gamma_{\mathbb{C}}|\boldsymbol{\sigma}|^{2} \quad \forall \boldsymbol{\sigma} \in \mathbb{R}_{\mathrm{sym}}^{2 \times 2} \text { and f.a.a. } x \in \Omega .
$$

For isotropic materials, the material is completely described by the two Lamé parameters $\lambda_{L}$ and $\mu_{L}$ which depend on Poisson's ratio $\nu$ and Young's modulus E. Hooke's law states that the stress $\sigma$ depends linearly on the strain $\varepsilon$

$$
\boldsymbol{\sigma}=\mathbb{C} \varepsilon=\lambda_{L} \operatorname{trace}(\varepsilon) \boldsymbol{I}+2 \mu_{L} \varepsilon .
$$

While general results regarding the analysis of our system as well as the error estimation are independent of the specific material, for the numerics we will work with isotropic materials. Apart from aspects regarding implementation, this choice also has the advantage that regularity results are obtained more easily.

The initial state $d_{0}$ is, unless otherwise stated, a function in $L^{2}(\Omega)$. The applied volume and boundary forces $l$ should belong to $H^{1}\left(I ; \boldsymbol{L}^{2}(\Omega)\right)$ which compactly embeds into $L^{\infty}\left(I ; \boldsymbol{W}^{-1, p}(\Omega)\right)$ for all $2<p<\infty$. The parameter $p$ will be specified later.

For arbitrary $p \in[1, \infty]$ we define the subset $\boldsymbol{W}_{D}^{1, p}(\Omega)$ of $\boldsymbol{W}^{1, p}(\Omega)$ as

$$
\boldsymbol{W}_{D}^{1, p}(\Omega):=\left\{u \in \boldsymbol{W}^{1, p}(\Omega): u_{\mid \Gamma_{D}}=0\right\} .
$$

The dual space of $\boldsymbol{W}_{D}^{1, p^{\prime}}(\Omega)$ will be denoted by $\boldsymbol{W}^{-1, p}(\Omega)$ with $p^{\prime}$ being the conjugate exponent to $p$.

Let us state some results regarding the max-operator. While the first three results are well known, a proof for the last result may be found in Section 5.4 of [31], for a similar result about the absolute value function which is equivalent to the max-operator.

Lemma 2.2. (i) The Nemytskii-operators $\max : L^{2}(\Omega) \rightarrow L^{2}(\Omega)$, $\max : L^{\infty}(\Omega) \rightarrow L^{\infty}(\Omega)$ associated to $\max : \mathbb{R} \rightarrow \mathbb{R}, \max (y)=\max \{y, 0\}$, are well-defined and globally Lipschitz continuous with Lipschitz constant 1.

(ii) The Nemytskii-operators $\max : L^{2}\left(I ; L^{2}(\Omega)\right) \rightarrow L^{2}\left(I ; L^{2}(\Omega)\right)$, $\max : L^{2}\left(I ; L^{\infty}(\Omega)\right) \rightarrow L^{2}\left(I ; L^{\infty}(\Omega)\right)$ are well-defined and globally Lipschitz continuous with Lipschitz constant 1.

(iii) For arbitrary $v, r \in \mathbb{R}, r>0$ we have $\max (v-r) \leq \max (v) \leq|v|$. This inequality also holds true for $v \in L^{2}(\Omega)$ and $r \in \mathbb{R}, r>0$, that is we have

$$
\|\max (v-r)\|_{L^{2}(\Omega)} \leq\|\max (v)\|_{L^{2}(\Omega)} \leq\|v\|_{L^{2}(\Omega)} .
$$

(iv) The Nemytskii-operator $\max : H^{s}(\Omega) \rightarrow H^{s}(\Omega)$ is well-defined if and only if $0 \leq s<\frac{3}{2}$. For $y \in H^{s}(\Omega)$, we have

$$
\|\max (y)\|_{H^{s}(\Omega)} \leq C\|y\|_{H^{s}(\Omega)} .
$$

We use the following short notation for inner products and norms on $L^{2}(\Omega)$ and $L^{2}\left(I ; L^{2}(\Omega)\right)$ :

$$
\begin{array}{rlrl}
(v, w) & :=(v, w)_{L^{2}(\Omega)}, & (v, w)_{I \times \Omega}:=(v, w)_{L^{2}\left(I ; L^{2}(\Omega)\right)}, \\
\|v\|:=\|v\|_{L^{2}(\Omega)}, & \|v\|_{I \times \Omega}:=\|v\|_{L^{2}\left(I ; L^{2}(\Omega)\right)} .
\end{array}
$$

The duality product for $v \in \boldsymbol{W}^{-1, p}(\Omega)$ and $w \in \boldsymbol{W}_{D}^{1, p^{\prime}}(\Omega)$ is indicated by $\langle v, w\rangle$ and the time-dependent variant for $v \in L^{2}\left(I ; \boldsymbol{W}^{-1, p}(\Omega)\right), w \in L^{2}\left(I ; \boldsymbol{W}_{D}^{1, p^{\prime}}(\Omega)\right)$ is defined as $\langle v, w\rangle_{I \times \Omega}:=\int_{I}\langle v(t), w(t)\rangle \mathrm{d} t$.

Instead of the formulation (1.1)-(1.4) we will work with an alternative formulation of the problem. As $\operatorname{div}: L^{p}\left(\Omega ; \mathbb{R}_{\mathrm{sym}}^{2 \times 2}\right) \rightarrow \boldsymbol{W}^{-1, p}(\Omega)$ denotes the distributional vector-valued divergence, that is

$$
\langle\operatorname{div} \boldsymbol{\sigma}, v\rangle:=-\int_{\Omega} \boldsymbol{\sigma}: \varepsilon(v) \mathrm{d} x, \quad \forall v \in \boldsymbol{W}_{D}^{1, p^{\prime}}(\Omega)
$$


and $\Delta: \boldsymbol{W}^{1, p}(\Omega) \rightarrow \boldsymbol{W}^{1, p^{\prime}}(\Omega)$ is the distributional Laplace-operator, i.e., $\mathrm{s}$

$$
\langle\Delta \phi, \psi\rangle:=-\int_{\Omega} \nabla \phi \nabla \psi \mathrm{d} x \quad \forall \psi \in \boldsymbol{W}^{1, p^{\prime}}(\Omega)
$$

we may alternatively express the damage model as

$$
\begin{aligned}
(g(\varphi) \mathbb{C} \varepsilon(\boldsymbol{u}), \varepsilon(v))_{I \times \Omega} & =\langle l, v\rangle_{I \times \Omega} & & \forall v \in L^{2}\left(I ; \boldsymbol{W}_{D}^{1, p^{\prime}}(\Omega)\right) \\
-\alpha(\nabla \varphi, \nabla \psi)_{I \times \Omega}+\beta(\varphi, \psi)_{I \times \Omega}+\frac{1}{2}\left(g^{\prime}(\varphi) \mathbb{C} \varepsilon(\boldsymbol{u}): \varepsilon(\boldsymbol{u}), \psi\right)_{I \times \Omega} & =\beta(d, \psi)_{I \times \Omega} & & \forall \psi \in L^{2}\left(I ; H^{1}(\Omega)\right) \\
\left(\partial_{t} d, \lambda\right)_{I \times \Omega}-\frac{1}{\delta}(\max (-\beta(d-\varphi)-r), \lambda)_{I \times \Omega} & =0 & & \forall \lambda \in L^{2}\left(I ; L^{2}(\Omega)\right) \\
d(0) & =d_{0} & &
\end{aligned}
$$

Next, we briefly summarize all known results about the continuous damage model (1.1)-(1.4) which we will need in the following sections. All of them can be found in [25,33] or in a more detailed way in [32].

We start with a time-independent variant of the PDE-system (1.1)-(1.2).

Definition 2.3 (see [25], Def. 3.2). For given $\varphi \in L^{1}(\Omega)$, we define the linear form $A_{\varphi}: \boldsymbol{W}_{D}^{1,2}(\Omega) \rightarrow \boldsymbol{W}^{-1,2}(\Omega)$ as

$$
\left\langle A_{\varphi} \boldsymbol{u}, v\right\rangle:=\int_{\Omega} g(\varphi) \mathbb{C} \varepsilon(\boldsymbol{u}): \varepsilon(v) \mathrm{d} x .
$$

Then, there exists a $p>2$ such that for all $r \in\left[p^{\prime}, p\right]$ and all $\varphi \in L^{1}(\Omega)$ the operator $A_{\varphi}: \boldsymbol{W}_{D}^{1, r}(\Omega) \rightarrow \boldsymbol{W}^{-1, r}(\Omega)$ is continuously invertible. Moreover, there exists a constant $c>0$ independent of $\varphi$ and $r$ such that

$$
\left\|A_{\varphi}^{-1} h\right\|_{\boldsymbol{W}_{D}^{1, r}(\Omega)} \leq c\|h\|_{\boldsymbol{W}^{-1, r}(\Omega)}
$$

holds true for all $h \in \boldsymbol{W}^{-1, r}(\Omega)$, for all $r \in\left[p^{\prime}, p\right]$ and for all $\varphi \in L^{1}(\Omega)$ (see [25], Lem. 3.3, [32], Rem. 1.4). For the rest of this chapter, we fix a $p>2$ which fulfills the previous result. With $A_{\varphi}$ at hand, we may now define the solution operator of the first PDE.

Definition 2.4 (see [25], Def. 3.6). We define the mapping $\mathcal{U}: \boldsymbol{W}^{-1, p}(\Omega) \times H^{1}(\Omega) \rightarrow \boldsymbol{W}_{D}^{1, p}(\Omega)$ as the solution operator of the time-independent variant of (1.1) which is given by

$$
\boldsymbol{u}:=\mathcal{U}(l, \varphi):=A_{\varphi}^{-1} l .
$$

For given $r \in[2 p /(p-2), \infty]$ and $\rho>0$, there exists an $L_{\mathcal{U}}=L_{\mathcal{U}}(\rho)>0$ such that

$$
\left\|\mathcal{U}\left(l_{1}, \varphi_{1}\right)-\mathcal{U}\left(l_{2}, \varphi_{2}\right)\right\|_{W_{D}^{1, \zeta}(\Omega)} \leq L_{\mathcal{U}}(\rho)\left(\left\|l_{1}-l_{2}\right\|_{W^{-1, p}(\Omega)}+\left\|\varphi_{1}-\varphi_{2}\right\|_{L^{r}(\Omega)}\right)
$$

with $\frac{1}{\zeta}=\frac{1}{p}+\frac{1}{r}$ for all $l_{1}, l_{2} \in \mathcal{B}_{\rho}:=\left\{l \in \boldsymbol{W}^{-1, p}(\Omega):\|l\|_{\boldsymbol{W}^{-1, p}(\Omega)}<\rho\right\}$ and for all $\varphi_{1}, \varphi_{2} \in H^{1}(\Omega) \cap L^{r}(\Omega)$. This means, that the solution operator $\mathcal{U}$ is Lipschitz continuous with respect to $l$ only in an open ball of radius $\rho$ (see [25], Prop. 3.8, [33], Lem. 3.1, [32], p. 95f). For a fixed $l \in L^{\infty}\left(I ; \boldsymbol{W}^{-1, p}(\Omega)\right.$ ), we may just set $\rho:=\|l\|_{L^{\infty}\left(I ; \boldsymbol{W}^{-1, p}(\Omega)\right)}$. Next, we deal with unique solvability of the time-independent variant of (1.2).

Definition 2.5 (see [25], Def. 3.13). We define the mappings $B: H^{1}(\Omega) \rightarrow H^{1}(\Omega)^{*}$ and $F: \boldsymbol{W}^{-1, p}(\Omega) \times H^{1}(\Omega) \rightarrow$ $H^{1}(\Omega)^{*}$ as

$$
\langle B \varphi, \psi\rangle:=\int_{\Omega} \alpha \nabla \varphi \nabla \psi+\beta \varphi \psi \mathrm{d} x
$$

and

$$
\langle F(l, \varphi), \psi\rangle:=\frac{1}{2} \int_{\Omega} g^{\prime}(\varphi) \mathbb{C} \varepsilon(\mathcal{U}(l, \varphi)): \varepsilon(\mathcal{U}(l, \varphi)) \psi \mathrm{d} x .
$$


We will make use of two properties of the mapping $F$. The first is Lipschitz continuity with respect to $\varphi$ for fixed $l$ (see [25], Lem. 3.17, [32], p. 98 for a slightly more general result):

$$
\left|\left\langle F\left(l, \varphi_{1}\right)-F\left(l, \varphi_{2}\right), \psi\right\rangle\right| \leq C\|l\|_{W^{-1, p}(\Omega)}^{2}\left\|\varphi_{1}-\varphi_{2}\right\|_{L^{r}(\Omega)}\|\psi\|_{L^{r}(\Omega)}
$$

which holds true for all $\varphi_{1}, \varphi_{2}, \psi \in H^{1}(\Omega)$ and for $r \geq 2 p /(p-2)$. The second one is strong monotonicity of the operator $B+F$ under Assumption 2.1 (see [25], Lem. 3.19, [32], p. 99):

$$
\left\langle B\left(\varphi_{1}-\varphi_{2}\right)+F\left(l, \varphi_{1}\right)-F\left(l, \varphi_{2}\right), \varphi_{1}-\varphi_{2}\right\rangle \geq \frac{\alpha}{2}\left\|\varphi_{1}-\varphi_{2}\right\|_{H^{1}(\Omega)}^{2}
$$

holds true for all $\varphi_{1}, \varphi_{2} \in H^{1}(\Omega)$, provided that $\beta$ is chosen large enough.

Definition 2.6 (see [25], Def. 3.21, [33], Lem. 3.1). We define the operator $\Phi: \mathcal{B}_{\rho} \times L^{2}(\Omega) \rightarrow H^{1}(\Omega)$ as

$$
\varphi=\Phi(l, d):=(B+F(l, \cdot))^{-1}(\beta d) .
$$

$\Phi$ is Lipschitz continuous for all $l_{1}, l_{2} \in \mathcal{B}_{\rho}$ and all $d_{1}, d_{2} \in L^{2}(\Omega)$ with a constant $L_{\Phi}=L_{\Phi}(\rho)>0$

$$
\left\|\Phi\left(l_{1}, d_{1}\right)-\Phi\left(l_{2}, d_{2}\right)\right\|_{H^{1}(\Omega)} \leq L_{\Phi}\left\{\left\|l_{1}-l_{2}\right\|_{W^{-1, p}(\Omega)}+\left\|d_{1}-d_{2}\right\|\right\} .
$$

With both time-independent solution operators at hand, one can reduce the ODE (2.5) onto the variable $d$. Existence of a unique solution $d \in W^{1, \infty}\left(I ; L^{2}(\Omega)\right)$ for given $l \in L^{\infty}\left(I ; \boldsymbol{W}^{-1, p}(\Omega)\right)$ can then be shown via a contraction argument.

Theorem 2.7 (see [33], Def. 3.4). Let $\rho>0$ be given and Assumption 2.1 be fulfilled. For an initial value $d_{0} \in L^{2}(\Omega)$ and a load $l \in \mathcal{B}_{\rho}^{\infty}:=\left\{l \in L^{\infty}\left(I ; \boldsymbol{W}^{-1, p}(\Omega)\right):\|l\|_{L^{\infty}\left(I ; \boldsymbol{W}^{-1, p}(\Omega)\right)}<\rho\right\}$, there exists a unique solution $(\boldsymbol{u}, \varphi, d) \in L^{\infty}\left(I ; \boldsymbol{W}_{D}^{1, p}(\Omega)\right) \times L^{\infty}\left(I ; H^{1}(\Omega)\right) \times W^{1, \infty}\left(I ; L^{2}(\Omega)\right)$ of the damage model (2.3)-(2.6) with $\boldsymbol{u}=\mathcal{U}(l(\cdot), \Phi(l(\cdot), d(\cdot)))$ and $\varphi=\Phi(l(\cdot), d(\cdot))$.

\section{Fréchet derivatives of $\mathcal{U}$ and $F$}

For the estimation of the spatial discretization error, we will need Lipschitz continuity of the Fréchet derivatives of $\mathcal{U}$ and $F$ with respect to $\varphi$. Therefore, we have a look at partial derivatives.

Lemma 2.8. There exists an index $\mu \in(2, p)$ such that for every $l \in \boldsymbol{W}^{-1, p}(\Omega)$ the map $\mathcal{U}(l, \cdot): H^{1}(\Omega) \rightarrow$ $\boldsymbol{W}_{D}^{1, \mu}(\Omega)$ is Fréchet differentiable and, for all $\varphi, \delta \varphi \in H^{1}(\Omega)$, the partial derivative fulfills

$$
A_{\varphi}\left(\partial_{\varphi} \mathcal{U}(l, \varphi) \delta \varphi\right)=\operatorname{div}\left(g^{\prime}(\varphi) \delta \varphi \mathbb{C} \varepsilon(\mathcal{U}(l, \varphi))\right) \quad \operatorname{in} \boldsymbol{W}^{-1, \mu}(\Omega)
$$

with div denoting the distributional divergence. Moreover, for $r \in[2 p /(p-2), 2 p \mu /(p-\mu)]$, we have

$$
\left\|\left[\partial_{\varphi} \mathcal{U}\left(l, \varphi_{1}\right)-\partial_{\varphi} \mathcal{U}\left(l, \varphi_{2}\right)\right] z\right\|_{W_{D}^{1, \nu}(\Omega)} \leq C(\rho)\left\|\varphi_{1}-\varphi_{2}\right\|_{L^{r}(\Omega)}\|z\|_{L^{r}(\Omega)}
$$

with $\frac{1}{\nu}=\frac{2}{r}+\frac{1}{p}$ for all $\varphi_{1}, \varphi_{2}, z \in H^{1}(\Omega) \cap L^{r}(\Omega)$.

Proof. We will only prove equation (2.12) as the first part has already been established in Lemma 5.3 of [25] and [32], p. 101. Due to the definition of the partial derivative w.r.t. $\varphi$ we have for arbitrary $\varphi_{1}, \varphi_{2}, z \in H^{1}(\Omega)$

$$
\begin{aligned}
& A_{\varphi_{1}}\left(\partial_{\varphi} \mathcal{U}\left(l, \varphi_{1}\right) z-\partial_{\varphi} \mathcal{U}\left(l, \varphi_{2}\right) z\right) \\
& \quad=\operatorname{div}\left(g^{\prime}\left(\varphi_{1}\right) z \mathbb{C} \varepsilon\left(\mathcal{U}\left(l, \varphi_{1}\right)\right)\right)-\operatorname{div}\left(g^{\prime}\left(\varphi_{2}\right) z \mathbb{C} \varepsilon\left(\mathcal{U}\left(l, \varphi_{2}\right)\right)\right)+\left(A_{\varphi_{2}}-A_{\varphi_{1}}\right)\left(\partial_{\varphi} \mathcal{U}\left(l, \varphi_{2}\right) z\right) .
\end{aligned}
$$


We will estimate both terms separately. We test the first two terms with $v \in \boldsymbol{W}_{D}^{1, \nu^{\prime}}(\Omega)$ to obtain

$$
\begin{aligned}
& \left.\int_{\Omega} g^{\prime}\left(\varphi_{1}\right) z \mathbb{C} \varepsilon\left(\mathcal{U}\left(l, \varphi_{1}\right)\right): \varepsilon(v)-g^{\prime}\left(\varphi_{2}\right) z \mathbb{C} \varepsilon\left(\mathcal{U}\left(l, \varphi_{2}\right)\right)\right): \varepsilon(v) \mathrm{d} x \\
& \quad=\int_{\Omega}\left[g^{\prime}\left(\varphi_{1}\right)-g^{\prime}\left(\varphi_{2}\right)\right] z \mathbb{C} \varepsilon\left(\mathcal{U}\left(l, \varphi_{1}\right)\right): \varepsilon(v) \mathrm{d} x+\int_{\Omega} g^{\prime}\left(\varphi_{2}\right) z \mathbb{C} \varepsilon\left(\mathcal{U}\left(l, \varphi_{1}\right)-\mathcal{U}\left(l, \varphi_{2}\right)\right): \varepsilon(v) \mathrm{d} x \\
& \quad \leq C\left\{\left\|\varphi_{1}-\varphi_{2}\right\|_{L^{r}(\Omega)}+\left\|\mathcal{U}\left(l, \varphi_{1}\right)-\mathcal{U}\left(l, \varphi_{2}\right)\right\|_{W_{D}^{1, \zeta}(\Omega)}\right\}\|z\|_{L^{r}(\Omega)}\|v\|_{W_{D}^{1, \nu^{\prime}}(\Omega)} \\
& \quad \leq C\left\|\varphi_{1}-\varphi_{2}\right\|_{L^{r}(\Omega)}\|z\|_{L^{r}(\Omega)}\|v\|_{W_{D}^{1, \nu^{\prime}}(\Omega)}
\end{aligned}
$$

where we employed the Lipschitz continuity of $g^{\prime},\left\|\mathcal{U}\left(l, \varphi_{1}\right)\right\|_{W_{D}^{1, p}(\Omega)} \leq C$ according to (2.7) for fixed $l$ and Hölder's inequality with $\frac{1}{\nu}=\frac{2}{r}+\frac{1}{p}$ and $\frac{1}{\nu}=\frac{1}{r}+\frac{1}{\zeta}$ which leads to $\frac{1}{\zeta}=\frac{1}{r}+\frac{1}{p}$ for the second to last estimate. For the last estimate, we made use of the Lipschitz continuity of $\mathcal{U}$ as $\zeta$ fulfills the condition, see (2.8). Thus, we obtain

$$
\left\|\operatorname{div}\left(g^{\prime}\left(\varphi_{1}\right) z \mathbb{C} \varepsilon\left(\mathcal{U}\left(l, \varphi_{1}\right)\right)\right)-\operatorname{div}\left(g^{\prime}\left(\varphi_{2}\right) z \mathbb{C} \varepsilon\left(\mathcal{U}\left(l, \varphi_{2}\right)\right)\right)\right\|_{\boldsymbol{W}^{-1, \nu}(\Omega)} \leq C\left\|\varphi_{1}-\varphi_{2}\right\|_{L^{r}(\Omega)}\|z\|_{L^{r}(\Omega)} .
$$

For the second term, we start with an upper bound for $\partial_{\varphi} \mathcal{U}\left(l, \varphi_{2}\right) z$. Hölder's inequality yields

$$
\left.\int_{\Omega} g^{\prime}\left(\varphi_{2}\right) z \mathbb{C} \varepsilon\left(\mathcal{U}\left(l, \varphi_{2}\right)\right)\right): \varepsilon(v) \mathrm{d} x \leq\left\|g^{\prime}\left(\varphi_{2}\right)\right\|_{L^{\infty}(\Omega)}\left\|\mathcal{U}\left(l, \varphi_{2}\right)\right\|_{W_{D}^{1, p}(\Omega)}\|z\|_{L^{r}(\Omega)}\|v\|_{W_{D}^{1, \zeta^{\prime}}(\Omega)}
$$

for $\frac{1}{\zeta}=\frac{1}{r}+\frac{1}{p}$. Thus we have

$$
\left\|\partial_{\varphi} \mathcal{U}\left(l, \varphi_{2}\right) z\right\|_{\boldsymbol{W}_{D}^{1, \zeta}(\Omega)} \leq C\left\|\operatorname{div}\left(g^{\prime}\left(\varphi_{2}\right) z \mathbb{C} \varepsilon\left(\mathcal{U}\left(l, \varphi_{2}\right)\right)\right)\right\|_{\boldsymbol{W}^{-1, \zeta}(\Omega)} \leq C\|z\|_{L^{r}(\Omega)} .
$$

since $r \geq \frac{2 p}{p-2}$ implies $\zeta \in[2, p]$ and (2.7) is valid. Next, we have for $\frac{1}{\nu}=\frac{1}{\zeta}+\frac{1}{r}=\frac{2}{r}+\frac{1}{p}$

$$
\begin{aligned}
& \int_{\Omega} g\left(\varphi_{2}\right) \mathbb{C} \varepsilon\left(\partial_{\varphi} \mathcal{U}\left(l, \varphi_{2}\right) z\right): \varepsilon(v)-g\left(\varphi_{1}\right) \mathbb{C} \varepsilon\left(\partial_{\varphi} \mathcal{U}\left(l, \varphi_{2}\right) z\right): \varepsilon(v) \mathrm{d} x \\
& \quad \leq\left\|g\left(\varphi_{2}\right)-g\left(\varphi_{1}\right)\right\|_{L^{r}(\Omega)}\left\|\partial_{\varphi} \mathcal{U}\left(l, \varphi_{2}\right) z\right\|_{\boldsymbol{W}_{D}^{1, \zeta}(\Omega)}\|v\|_{\boldsymbol{W}_{D}^{1, \nu^{\prime}}(\Omega)} \\
& \quad \leq C\left\|\varphi_{1}-\varphi_{2}\right\|_{L^{r}(\Omega)}\|z\|_{L^{r}(\Omega)}\|v\|_{\boldsymbol{W}_{D}^{1, \nu^{\prime}}(\Omega)} .
\end{aligned}
$$

This gives us

$$
\left\|\left(A_{\varphi_{2}}-A_{\varphi_{1}}\right)\left(\partial_{\varphi} \mathcal{U}\left(l, \varphi_{2}\right) z\right)\right\|_{\boldsymbol{W}^{-1, \nu}(\Omega)} \leq C(\rho)\left\|\varphi_{1}-\varphi_{2}\right\|_{L^{r}(\Omega)}\|z\|_{L^{r}(\Omega)} .
$$

By combining these two estimates (2.13) and (2.14), we arrive at

$$
\left\|A_{\varphi_{1}}\left(\partial_{\varphi} \mathcal{U}\left(l, \varphi_{1}\right) z-\partial_{\varphi} \mathcal{U}\left(l, \varphi_{2}\right) z\right)\right\|_{W^{-1, \nu}(\Omega)} \leq C\left\|\varphi_{1}-\varphi_{2}\right\|_{L^{r}(\Omega)}\|z\|_{L^{r}(\Omega)} .
$$

Again due to $r \geq \frac{2 p}{p-2}$, we have $\nu \geq p^{\prime}$ and (2.7) finally leads to

$$
\left\|\left[\partial_{\varphi} \mathcal{U}\left(l, \varphi_{1}\right)-\partial_{\varphi} \mathcal{U}\left(l, \varphi_{2}\right)\right] z\right\|_{W_{D}^{1, \nu}(\Omega)} \leq C\left\|\varphi_{1}-\varphi_{2}\right\|_{L^{r}(\Omega)}\|z\|_{L^{r}(\Omega)} .
$$

Lemma 2.9. For every $l \in \boldsymbol{W}^{-1, p}(\Omega)$, the function $F(l, \cdot): H^{1}(\Omega) \rightarrow H^{1}(\Omega)^{*}$ is Fréchet differentiable and its derivative at $\varphi \in H^{1}(\Omega)$ in direction $\delta \varphi \in H^{1}(\Omega)$ is given as

$$
\begin{aligned}
\left\langle\partial_{\varphi} F(l, \varphi) \delta \varphi, \psi\right\rangle= & \frac{1}{2} \int_{\Omega} g^{\prime \prime}(\varphi) \delta \varphi \mathbb{C} \varepsilon(\mathcal{U}(l, \varphi)): \varepsilon(\mathcal{U}(l, \varphi)) \psi \mathrm{d} x \\
& +\int_{\Omega} g^{\prime}(\varphi) \mathbb{C} \varepsilon(\mathcal{U}(l, \varphi)): \varepsilon\left(\partial_{\varphi} \mathcal{U}(l, \varphi) \delta \varphi\right) \psi \mathrm{d} x
\end{aligned}
$$


Moreover, we have for $r \in[3 p /(p-2), 2 p \mu /(p-\mu)]$ with $\mu \in(2, p)$ as in the previous lemma and $\varphi_{1}, \varphi_{2}, z, \psi \in$ $H^{1}(\Omega) \cap L^{r}(\Omega)$

$$
\left\langle\left[\partial_{\varphi} F\left(l, \varphi_{1}\right)-\partial_{\varphi} F\left(l, \varphi_{2}\right)\right] z, \psi\right\rangle \leq C(\rho)\left\|\varphi_{1}-\varphi_{2}\right\|_{L^{r}(\Omega)}\|z\|_{L^{r}(\Omega)}\|\psi\|_{L^{r}(\Omega)} .
$$

Proof. Again, the first part of the lemma has already been proven in Lemma 5.9 of [25] and [32], p. 105. Thus, we only prove the Lipschitz continuity (2.16). According to the definition of the partial derivative w.r.t. $\varphi$, we have

$$
\begin{aligned}
\left\langle\left[\partial_{\varphi}\right.\right. & \left.\left.F\left(l, \varphi_{1}\right)-\partial_{\varphi} F\left(l, \varphi_{2}\right)\right] z, \psi\right\rangle \\
= & \frac{1}{2} \int_{\Omega}\left(g^{\prime \prime}\left(\varphi_{1}\right)-g^{\prime \prime}\left(\varphi_{2}\right)\right) \mathbb{C} \varepsilon\left(\mathcal{U}\left(l, \varphi_{1}\right)\right): \varepsilon\left(\mathcal{U}\left(l, \varphi_{1}\right)\right) z \psi \mathrm{d} x \\
& +\frac{1}{2} \int_{\Omega} g^{\prime \prime}\left(\varphi_{2}\right)\left[\mathbb{C} \varepsilon\left(\mathcal{U}\left(l, \varphi_{1}\right)\right): \varepsilon\left(\mathcal{U}\left(l, \varphi_{1}\right)\right)-\mathbb{C} \varepsilon\left(\mathcal{U}\left(l, \varphi_{2}\right)\right): \varepsilon\left(\mathcal{U}\left(l, \varphi_{2}\right)\right)\right] z \psi \mathrm{d} x \\
& +\int_{\Omega}\left(g^{\prime}\left(\varphi_{1}\right)-g^{\prime}\left(\varphi_{2}\right)\right) \mathbb{C} \varepsilon\left(\mathcal{U}\left(l, \varphi_{1}\right)\right): \varepsilon\left(\partial_{\varphi} \mathcal{U}\left(l, \varphi_{1}\right) z\right) \psi \mathrm{d} x \\
& +\int_{\Omega} g^{\prime}\left(\varphi_{2}\right)\left[\mathbb{C} \varepsilon\left(\mathcal{U}\left(l, \varphi_{1}\right)\right): \varepsilon\left(\partial_{\varphi} \mathcal{U}\left(l, \varphi_{1}\right) z\right)-\mathbb{C} \varepsilon\left(\mathcal{U}\left(l, \varphi_{2}\right)\right): \varepsilon\left(\partial_{\varphi} \mathcal{U}\left(l, \varphi_{2}\right) z\right)\right] \psi \mathrm{d} x \\
= & (\mathrm{I})+(\mathrm{II})+(\mathrm{III})+(\mathrm{IV}) .
\end{aligned}
$$

We will estimate all four terms separately. For the first term, Hölder's inequality with $1=\frac{2}{p}+\frac{3}{r},(2.7)$ and Lipschitz continuity of $g^{\prime \prime}$ yield

$$
\text { (I) } \leq C\left\|\varphi_{1}-\varphi_{2}\right\|_{L^{r}(\Omega)}\|z\|_{L^{r}(\Omega)}\|\psi\|_{L^{r}(\Omega)} .
$$

Note, that $C$ depends on $\|l\|_{\boldsymbol{W}^{-1, p}(\Omega)}$ according to (2.7). For the second term, we have

$$
(\mathrm{II}) \leq\left\|g^{\prime \prime}\left(\varphi_{2}\right)\right\|_{L^{\infty}(\Omega)}\left\|\mathcal{U}\left(l, \varphi_{1}\right)+\mathcal{U}\left(l, \varphi_{2}\right)\right\|_{W_{D}^{1, p}(\Omega)}\left\|\mathcal{U}\left(l, \varphi_{1}\right)-\mathcal{U}\left(l, \varphi_{2}\right)\right\|_{W_{D}^{1, \zeta}(\Omega)}\|z\|_{L^{r}(\Omega)}\|\psi\|_{L^{r}(\Omega)}
$$

with $\frac{1}{\zeta}=\frac{1}{p}+\frac{1}{r}$ and $1=\frac{1}{\zeta}+\frac{1}{p}+\frac{2}{r}=\frac{2}{p}+\frac{3}{r}$. Lipschitz continuity of $\mathcal{U}$ then gives us

$$
\text { (II) } \leq C\left\|\varphi_{1}-\varphi_{2}\right\|_{L^{r}(\Omega)}\|z\|_{L^{r}(\Omega)}\|\psi\|_{L^{r}(\Omega)} .
$$

We also apply Hölder's inequality for the third term to arrive at

$$
(\mathrm{III}) \leq\left\|\varphi_{1}-\varphi_{2}\right\|_{L^{r}(\Omega)}\left\|\mathcal{U}\left(l, \varphi_{1}\right)\right\|_{W_{D}^{1, p}(\Omega)}\left\|\partial_{\varphi} \mathcal{U}\left(l, \varphi_{1}\right) z\right\|_{W_{D}^{1, \zeta}(\Omega)}\|\psi\|_{L^{r}(\Omega)}
$$

with $\frac{1}{\zeta}=\frac{1}{p}+\frac{1}{r}$ and $1=\frac{1}{\zeta}+\frac{1}{p}+\frac{2}{r}=\frac{2}{p}+\frac{3}{r}$. Boundedness of $\left\|\partial_{\varphi} \mathcal{U}\left(l, \varphi_{1}\right) z\right\|_{W_{D}^{1, \zeta}(\Omega)} \leq C\|z\|_{L^{r}(\Omega)}$ has been shown in the previous proof. Therefore, we have

$$
(\mathrm{III}) \leq C\left\|\varphi_{1}-\varphi_{2}\right\|_{L^{r}(\Omega)}\|z\|_{L^{r}(\Omega)}\|\psi\|_{L^{r}(\Omega)} .
$$

For the last term, we estimate

$$
(\mathrm{IV}) \leq C\left\|\mathbb{C} \varepsilon\left(\mathcal{U}\left(l, \varphi_{1}\right)\right): \varepsilon\left(\partial_{\varphi} \mathcal{U}\left(l, \varphi_{1}\right) z\right)-\mathbb{C} \varepsilon\left(\mathcal{U}\left(l, \varphi_{2}\right)\right): \varepsilon\left(\partial_{\varphi} \mathcal{U}\left(l, \varphi_{2}\right) z\right)\right\|_{L^{\mu}(\Omega)}\|\psi\|_{L^{r}(\Omega)}
$$

with $1=\frac{1}{\mu}+\frac{1}{r}$. Application of the triangle inequality to the $L^{\mu}$-norm term yields

$$
\begin{aligned}
\| \mathbb{C} \varepsilon\left(\mathcal{U}\left(l, \varphi_{1}\right)\right): \varepsilon\left(\partial_{\varphi} \mathcal{U}\left(l, \varphi_{1}\right) z\right)- & \mathbb{C} \varepsilon\left(\mathcal{U}\left(l, \varphi_{2}\right)\right): \varepsilon\left(\partial_{\varphi} \mathcal{U}\left(l, \varphi_{2}\right) z\right) \|_{L^{\mu}(\Omega)} \\
\leq & C\left\|\mathcal{U}\left(l, \varphi_{1}\right)-\mathcal{U}\left(l, \varphi_{2}\right)\right\|_{\boldsymbol{W}_{D}^{1, \zeta}(\Omega)}\left\|\partial_{\varphi} \mathcal{U}\left(l, \varphi_{1}\right) z\right\|_{\boldsymbol{W}_{D}^{1, \zeta^{\prime}}(\Omega)} \\
& +C\left\|\mathcal{U}\left(l, \varphi_{2}\right)\right\|_{\boldsymbol{W}_{D}^{1, p}(\Omega)}\left\|\partial_{\varphi} \mathcal{U}\left(l, \varphi_{1}\right) z-\partial_{\varphi} \mathcal{U}\left(l, \varphi_{2}\right) z\right\|_{\boldsymbol{W}_{D}^{1, s}(\Omega)}
\end{aligned}
$$


with $\frac{1}{\mu}=\frac{1}{\zeta}+\frac{1}{\zeta^{\prime}}=\frac{1}{p}+\frac{1}{s}$. We choose $\zeta$ such that $\frac{1}{\zeta}=\frac{1}{p}+\frac{1}{r}$. Due to $1=\frac{1}{\mu}+\frac{1}{r}$, this leads to $\frac{1}{\zeta^{\prime}}=\frac{1}{p}+\frac{1}{r}$ as well. Therefore, we are allowed to use Lipschitz continuity of $\mathcal{U}$ as well as boundedness of $\partial_{\varphi} \mathcal{U}\left(l, \varphi_{1}\right) z$. Due to $\frac{1}{\mu}=\frac{1}{p}+\frac{1}{s}$, we have $\frac{1}{s}=\frac{1}{p}+\frac{2}{r}$. Thus, Lipschitz continuity of $\partial_{\varphi} \mathcal{U}(l, \cdot)$ is employable. Together, we arrive at

$$
(\mathrm{IV}) \leq C\left\|\varphi_{1}-\varphi_{2}\right\|_{L^{r}(\Omega)}\|z\|_{L^{r}(\Omega)}\|\psi\|_{L^{r}(\Omega)} .
$$

The estimates for (I) - (IV) then yield the required result.

For a Lipschitz continuous derivative, it is easy to show that we have

$$
\left\|F\left(l, \varphi_{2}\right)-F\left(l, \varphi_{1}\right)-\partial_{\varphi} F\left(l, \varphi_{1}\right)\left(\varphi_{2}-\varphi_{1}\right)\right\|_{H^{1}(\Omega)^{*}} \leq C\left\|\varphi_{1}-\varphi_{2}\right\|_{H^{1}(\Omega)}^{2} .
$$

Note further, that $\partial_{\varphi} F(l, \varphi)$ is symmetric, that is we have

$$
\left\langle\partial_{\varphi} F(l, \varphi) z, \psi\right\rangle=\left\langle\partial_{\varphi} F(l, \varphi) \psi, z\right\rangle
$$

for all $z, \psi \in H^{1}(\Omega)$.

\section{Regularity of solutions}

For the error estimation, the regularity given by Theorem 2.7 is not satisfying. On the one hand, we need more temporal regularity. We have to assume more temporal regularity for $l$ for the temporal error estimation, that is, we assume $l \in H^{1}\left(I ; \boldsymbol{L}^{2}(\Omega)\right) \hookrightarrow \hookrightarrow L^{\infty}\left(I ; \boldsymbol{W}^{-1, p}(\Omega)\right)$. This is due to the fact that the solution of the PDE system is only as regular in time as the right-hand side. On the other hand, we also require more spatial regularity for semidiscrete solutions. As these are solutions of time-independent elliptic equations we have a look at the regularity of solutions $\boldsymbol{u}$ and $\varphi$ given by the Definitions 2.4 and 2.6 next.

Higher regularity for solutions to elliptic systems in divergence form with mixed boundary conditions has been studied in a recent paper [18]. Without going into detail here (for details, we refer to [18], Sect.5), we require that $g(\varphi) \mathbb{C}$ is a multiplier on $H^{\omega}\left(\Omega, \mathbb{R}^{2}\right)$ for some $\omega>0$. A sufficient condition for this property is Hölder continuity according to Lemma 1 of [18].

Thus, we have a look at the regularity of $\varphi=\Phi(l, d)$ first. We assume $\boldsymbol{u} \in \boldsymbol{W}_{D}^{1, p}(\Omega)$ and $d \in H^{1}(\Omega)$ since according to Theorem 3.12 of [19], the semidiscrete solution $d$ is more regular in space. It is as regular as $\varphi$ (which at the moment and according to Theorem 2.7 belongs to $H^{1}(\Omega)$ ) as long as the regularity is less than $H^{\frac{3}{2}}(\Omega)$.

Proposition 2.10. Let $l \in \boldsymbol{L}^{2}(\Omega)$ and $d \in H^{1}(\Omega)$ be given. Then, the solution $\varphi=\Phi(l, d)$ given by Definition 2.6 belongs to $W^{2, \frac{p}{2}}(\Omega) \hookrightarrow C^{0, \sigma}(\Omega)$ and thus is Hölder continuous with Hölder exponent

$$
\sigma= \begin{cases}2-\frac{4}{p}, & \text { if } p<4 \\ 1, & \text { if } p>4 \\ 1-\varepsilon, & \text { if } p=4\end{cases}
$$

Moreover, it fulfills

$$
\|\varphi\|_{W^{2, \frac{p}{2}(\Omega)}} \leq C\left\{\|d\|_{L^{\frac{p}{2}(\Omega)}}+\|l\|_{L^{2}(\Omega)}^{2}\right\} .
$$

Proof. The proposition follows with standard argumentation from elliptic regularity theory.

Thus, we have Hölder continuity of $g(\varphi)$ since $\varphi$ is Hölder continuous and $g$ is Lipschitz continuous which yields that $g(\varphi)$ is indeed a multiplier. For $\mathbb{C}$, we could also impose Hölder continuity. But since $\mathbb{C}$ depends on material parameters, for which continuity might be to restrictive, we just assume the following

Assumption 2.11. From now on, we assume that $\mathbb{C}$ is a multiplier on $H^{\omega}\left(\Omega, \mathbb{R}^{2}\right)$ for some $\omega>0$. 
Note, that Remark 5 of [18], ensures that Assumption 2.11 is fulfilled for example for piecewise constant, discontinuous material parameter functions. Based on this assumption we arrive at the following regularity result

Lemma 2.12. With Assumption 2.11, for a given right-hand side $l \in L^{2}(\Omega)$ and a function $\varphi \in C^{0, \sigma}(\Omega)$, $\sigma \in(0,1)$ the solution of the equation

$$
-\operatorname{div}(g(\varphi) \mathbb{C} \varepsilon(\boldsymbol{u}))=l
$$

admits the additional regularity $u \in \boldsymbol{H}_{D}^{1+\kappa}(\Omega)$ for a fixed $\kappa \in(0,1)$ and fulfills

$$
\|\boldsymbol{u}\|_{\boldsymbol{H}_{D}^{1+\kappa}(\Omega)} \leq C\|l\|_{H^{\kappa-1}(\Omega)}
$$

with a constant $C>0$ depending on the multiplier norm of $\varphi$.

Remark 2.13. Lemma 2.12 only ensures the existence of $\kappa$ such that $\boldsymbol{u} \in \boldsymbol{H}_{D}^{1+\kappa}(\Omega)$ but the relation of $\kappa$ and the integrability $p>2$ obtained by $W^{1, p}$-theory is still an open question. For this contribution, we assume that the integrability obtained by $W^{1, p}$-theory cannot be improved any further. Since $\boldsymbol{u} \in \boldsymbol{H}_{D}^{1+\kappa}(\Omega) \hookrightarrow \boldsymbol{W}_{D}^{1, \frac{2}{1-\kappa}}(\Omega)$ we therefore assume that

$$
\kappa<1-\frac{2}{p}
$$

holds true for the largest possible $p$ obtained by $W^{1, p}$-theory. In case there is no largest possible $p$, the above inequality will be assumed for the supremum $\tilde{p} \in(2, \infty]$.

Based on Proposition 2.10 we state the improved regularity of $\varphi$

Lemma 2.14. Let Assumption 2.11 be fulfilled. Moreover, let $l \in \boldsymbol{L}^{2}(\Omega)$ and $d \in H^{1}(\Omega)$ be given. Then, the solution given by Definition 2.6 belongs to $W^{2, \frac{p}{2}}(\Omega) \hookrightarrow H^{1+\theta}(\Omega), \theta=\min \left\{1,2-\frac{4}{p}\right\}$ and fulfills

$$
\|\varphi\|_{H^{1+\theta}(\Omega)} \leq C\left\{\|d\|_{L^{2}(\Omega)}+\|l\|_{L^{2}(\Omega)}^{2}\right\}
$$

with a constant $C>0$ independent of $l$ and $d$.

Finally, to be able to apply duality arguments, we need to know the regularity of solutions to linearized equations. Since $A_{\varphi} \boldsymbol{u}=l$ is already linear in $\boldsymbol{u}$, we only have a look at solutions of

$$
\left\langle B z+\partial_{\varphi} F(l, \varphi) z, \psi\right\rangle=(f, \psi) \quad \forall \psi \in H^{1}(\Omega)
$$

with $\varphi=\Phi(l, d)$ for given $l \in L^{2}(\Omega), f \in L^{\nu}(\Omega)$.

Lemma 2.15. Let Assumptions 2.1 and 2.11 be fulfilled. Moreover, let $l \in L^{2}(\Omega), d \in L^{2}(\Omega), \varphi=\Phi(l, d)$ and $f \in L^{\nu}(\Omega)$ be given. Then, the variational problem

$$
\left\langle B z+\partial_{\varphi} F(l, \varphi) z, \psi\right\rangle=(f, \psi) \quad \forall \psi \in H^{1}(\Omega)
$$

possesses a unique solution $z \in H^{1}(\Omega) \cap W^{2, \omega}(\Omega)$ which fulfills

$$
\|z\|_{W^{2, \omega}(\Omega)} \leq C\|f\|_{L^{\nu}(\Omega)}
$$

with a constant $C>0$ only depending on $\|l\|_{L^{2}(\Omega)}$ and with $\omega=\min \left\{\nu, \frac{p}{2}\right\}$.

Proof. This is a standard result from elliptic theory.

\section{Semidiscretization in time}

Now, we turn our attention towards the a priori error estimates for a discrete version of the damage model. We split the errors in a temporal and a spatial part and start with the error in time. 


\subsection{Discretization in time}

For the discretization in time we will employ discontinuous constant finite elements. Therefore, we consider a partition of the time interval $\bar{I}=[0, T]$ as

$$
\bar{I}=\{0\} \cup I_{1} \cup \ldots \cup I_{M}
$$

with subintervals $I_{m}=\left(t_{m-1}, t_{m}\right]$ of length $\tau_{m}$ and time points

$$
0=t_{0}<t_{1}<\ldots<t_{M-1}<t_{M}=T .
$$

We set $\tau:=\max \left\{\tau_{m}: m=1, \ldots, M\right\}$. The semidiscrete trial and test spaces are given as

$$
\begin{aligned}
\boldsymbol{U}_{\tau}^{0}(p) & :=\left\{\boldsymbol{u}_{\tau} \in L^{2}\left(I ; \boldsymbol{W}_{D}^{1, p}(\Omega)\right): \boldsymbol{u}_{\left.\tau\right|_{I_{m}}} \in \mathbb{P}_{0}\left(I_{m} ; \boldsymbol{W}_{D}^{1, p}(\Omega)\right), m=1, \ldots, M\right\}, \\
V_{\tau}^{0} & :=\left\{v_{\tau} \in L^{2}\left(I ; H^{1}(\Omega)\right): v_{\left.\tau\right|_{I_{m}}} \in \mathbb{P}_{0}\left(I_{m} ; H^{1}(\Omega)\right), m=1, \ldots, M\right\} \\
X_{\tau}^{0} & :=\left\{d_{\tau} \in L^{2}\left(I ; L^{2}(\Omega)\right): d_{\left.\tau\right|_{I_{m}}} \in \mathbb{P}_{0}\left(I_{m} ; L^{2}(\Omega)\right), m=1, \ldots, M\right\} .
\end{aligned}
$$

We use the notation

$$
(v, w)_{I_{m} \times \Omega}:=(v, w)_{L^{2}\left(I_{m} ; L^{2}(\Omega)\right)} \quad \text { and } \quad\|v\|_{I_{m} \times \Omega}:=\|v\|_{L^{2}\left(I_{m} ; L^{2}(\Omega)\right)} .
$$

To express the jumps possibly occurring at the nodes $t_{m}$ we define

$$
v_{\tau, m}^{+}:=\lim _{t \rightarrow 0^{+}} v_{\tau}\left(t_{m}+t\right), \quad v_{\tau, m}^{-}:=\lim _{t \rightarrow 0^{+}} v_{\tau}\left(t_{m}-t\right)=v_{\tau}\left(t_{m}\right), \quad\left[v_{\tau}\right]_{m}=v_{\tau, m}^{+}-v_{\tau, m}^{-} .
$$

Note, that for functions piecewise constant in time the definition reduces to

$$
v_{\tau, m}^{+}=v_{\tau}\left(t_{m+1}\right)=: v_{\tau, m+1}, \quad v_{\tau, m}^{-}=v_{\tau}\left(t_{m}\right)=: v_{\tau, m}, \quad\left[v_{\tau}\right]_{m}=v_{\tau, m+1}-v_{\tau, m} .
$$

Then, the semidiscrete problem is given as follows: Find states $\left(\boldsymbol{u}_{\tau}, \varphi_{\tau}, d_{\tau}\right) \in \boldsymbol{U}_{\tau}^{0}(p) \times V_{\tau}^{0} \times X_{\tau}^{0}$ such that

$$
\begin{gathered}
\left(g\left(\varphi_{\tau}\right) \mathbb{C} \varepsilon\left(\boldsymbol{u}_{\tau}\right), \varepsilon(v)\right)_{I \times \Omega}=\langle l, v\rangle_{I \times \Omega} \\
-\alpha\left(\nabla \varphi_{\tau}, \nabla \psi\right)_{I \times \Omega}+\beta\left(\varphi_{\tau}, \psi\right)_{I \times \Omega}+\frac{1}{2}\left(g^{\prime}\left(\varphi_{\tau}\right) \mathbb{C} \varepsilon\left(\boldsymbol{u}_{\tau}\right): \varepsilon\left(\boldsymbol{u}_{\tau}\right), \psi\right)_{I \times \Omega}=\beta\left(d_{\tau}, \psi\right)_{I \times \Omega} \\
\sum_{m=1}^{M}\left(\partial_{t} d_{\tau}, \lambda\right)_{I_{m} \times \Omega}+\sum_{m=2}^{M}\left(\left[d_{\tau}\right]_{m-1}, \lambda_{m-1}^{+}\right)+\left(d_{\tau, 0}^{+}, \lambda_{0}^{+}\right)=\frac{1}{\delta}\left(\max \left(-\beta\left(d_{\tau}-\varphi_{\tau}\right)-r\right), \lambda\right)_{I \times \Omega}+\left(d_{0}, \lambda_{0}^{+}\right)
\end{gathered}
$$

is fulfilled for all $(v, \psi, \lambda) \in \boldsymbol{U}_{\tau}^{0}\left(p^{\prime}\right) \times V_{\tau}^{0} \times X_{\tau}^{0}$.

We require the interpolation/projection onto $\boldsymbol{U}_{\tau}^{0}, X_{\tau}^{0}$ and $V_{\tau}^{0}$, respectively. Therefore, we define the semidiscrete interpolation operator $I_{\tau}: C\left(\bar{I} ; L^{2}(\Omega)\right) \rightarrow X_{\tau}^{0}$ with $I_{\tau} d_{\left.\right|_{I_{m}}} \in \mathbb{P}_{0}\left(I_{m} ; L^{2}(\Omega)\right)$ via $\left(I_{\tau} d\right)\left(t_{m}\right)=d\left(t_{m}\right)$ for $m=$ $1, \ldots, M$. For the projection we employ the standard $L^{2}$-projection in time $P_{\tau}: L^{2}\left(I ; L^{2}(\Omega)\right) \rightarrow X_{\tau}^{0}$ given by $P_{\tau} \varphi_{I_{m}}:=\frac{1}{\tau_{m}} \int_{I_{m}} \varphi(t) \mathrm{d} t$. Both operators will always be denoted by the same symbols despite possibly different domains and ranges. Note, that integration in time preserves spatial regularity due to the definition of the Bochner integral, that is $P_{\tau} z$ has the same spatial regularity as the preimage $z$. Note furthermore, that the projection $P_{\tau}$ is $L^{s}(I ; X)$-stable for all $s \in[1, \infty]$ and any arbitrary Banach space $X$.

\subsection{Temporal error estimates}

We will prove linear convergence in time in suitable norms for the displacement as well as for the two damage variables. In accordance with our discretization technique, we may choose test functions vanishing outside the 
subinterval $I_{m}$. Then, the solution of (3.1)-(3.3) also fulfills

$$
\begin{aligned}
\left(g\left(\varphi_{\tau}\right) \mathbb{C} \varepsilon\left(\boldsymbol{u}_{\tau}\right), \varepsilon(v)\right)_{I_{m} \times \Omega}= & \langle l, v\rangle_{I_{m} \times \Omega} \\
& \alpha\left(\nabla \varphi_{\tau}, \nabla \psi\right)_{I_{m} \times \Omega}+\beta\left(\varphi_{\tau}, \psi\right)_{I_{m} \times \Omega} \\
+\frac{1}{2}\left(g^{\prime}\left(\varphi_{\tau}\right) \mathbb{C} \varepsilon\left(\boldsymbol{u}_{\tau}\right): \varepsilon\left(\boldsymbol{u}_{\tau}\right), \psi\right)_{I_{m} \times \Omega}= & \beta\left(d_{\tau}, \psi\right)_{I_{m} \times \Omega} \\
d_{\tau, m}-d_{\tau, m-1}= & \frac{\tau_{m}}{\delta}\left(\max \left(-\beta\left(d_{\tau, m}-\varphi_{\tau, m}\right)-r\right)\right. \\
d_{\tau, 0}: & =d_{0}
\end{aligned}
$$

for $v \in \boldsymbol{U}_{\tau}^{0}\left(p^{\prime}\right), \psi \in V_{\tau}^{0}$ and for all $m=1, \ldots, M$. This system is equivalent to

$$
\begin{aligned}
\left(g\left(\varphi_{\tau, m}\right) \mathbb{C} \varepsilon\left(u_{\tau, m}\right), \varepsilon\left(v_{m}\right)\right)= & \left\langle P_{\tau} l_{\mid I_{m}}, v_{m}\right\rangle \\
& \alpha\left(\nabla \varphi_{\tau, m}, \nabla \psi_{m}\right)+\beta\left(\varphi_{\tau, m}, \psi_{m}\right) \\
+\frac{1}{2}\left(g^{\prime}\left(\varphi_{\tau, m}\right) \mathbb{C} \varepsilon\left(u_{\tau, m}\right): \varepsilon\left(u_{\tau, m}\right), \psi_{m}\right)= & \beta\left(d_{\tau, m}, \psi_{m}\right) \\
d_{\tau, m}-d_{\tau, m-1} & =\frac{\tau_{m}}{\delta}\left(\max \left(-\beta\left(d_{\tau, m}-\varphi_{\tau, m}\right)-r\right)\right. \\
d_{\tau, 0}: & =d_{0}
\end{aligned}
$$

for $v_{m} \in \boldsymbol{W}_{D}^{1, p^{\prime}}(\Omega), \psi_{m} \in H^{1}(\Omega)$ and for all $m=1, \ldots, M$. Note, that $P_{\tau} l_{\left.\right|_{I_{m}}} \in \boldsymbol{W}^{-1, p}(\Omega)$ according to the preserved spatial regularity of a temporal projection. We easily see that the solution of the now time-independent elliptic PDE system is given as

$$
u_{\tau, m}=\mathcal{U}\left(P_{\tau} l_{\left.\right|_{I_{m}}}, \varphi_{\tau, m}\right) \in \boldsymbol{W}_{D}^{1, p}(\Omega) \quad \varphi_{\tau, m}=\Phi\left(P_{\tau} l_{\left.\right|_{I_{m}}}, d_{\tau, m}\right) \in H^{1}(\Omega) .
$$

Since the reduced fixed point equation

$$
d=F_{m}(d):=d_{\tau, m-1}+\frac{\tau_{m}}{\delta} \max \left(-\beta\left(d-\Phi\left(P_{\tau} l_{I_{m}}, d\right)\right)-r\right)
$$

is identical to the fixed point equation in [19] (up to a different solution operator $\Phi$ ), existence of a unique solution $d_{\tau, m} \in L^{2}(\Omega)$ can be proven as in Proposition 3.1 of [19], by means of Banach's fixed point theorem. We only have to assume that $\frac{\beta}{\delta} \tau_{m}\left(1+L_{\Phi}\right)<1$ holds true with $L_{\Phi}$ denoting the Lipschitz constant of $\Phi$ such that $F_{m}: L^{2}(\Omega) \rightarrow L^{2}(\Omega)$ is a contraction. Uniqueness of $d_{\tau, m}$ then leads to unique solutions $\varphi_{\tau, m}$ and $u_{\tau, m}$, meaning that the semidiscrete damage model (3.1)-(3.3) possesses a unique solution.

Theorem 3.1. For a given right-hand side $l \in L^{\infty}\left(I ; \boldsymbol{W}^{-1, p}(\Omega)\right)$ and a given initial value $d_{0} \in L^{2}(\Omega)$, there exists a unique solution $\left(\boldsymbol{u}_{\tau}, \varphi_{\tau}, d_{\tau}\right) \in \boldsymbol{U}_{\tau}^{0}(p) \times V_{\tau}^{0} \times X_{\tau}^{0}$ of the semidiscrete damage model (3.1)-(3.3) provided that $\tau$ is sufficiently small. Moreover, if $l \in H^{1}\left(I ; \boldsymbol{L}^{2}(\Omega)\right)$ and $d_{0} \in H^{\frac{3}{2}-\epsilon}(\Omega), \epsilon \in\left(0, \frac{1}{2}\right]$, the semidiscrete solution admits the additional regularity $\left(\boldsymbol{u}_{\tau}, \varphi_{\tau}, d_{\tau}\right) \in L^{\infty}\left(I ; \boldsymbol{H}_{D}^{1+\kappa}(\Omega)\right) \times L^{\infty}\left(I ; H^{1+\theta}(\Omega)\right) \times L^{\infty}\left(I ; H^{s}(\Omega)\right)$ with $\kappa \in(0,1)$ given as in Lemma 2.12, $\theta=\min \left\{1,2-\frac{4}{p}\right\}$ and $s=\min \left\{1+\theta, \frac{3}{2}-\epsilon\right\}$ and the stability estimates

$$
\begin{aligned}
&\left\|\boldsymbol{u}_{\tau}\right\|_{L^{\infty}\left(I ; \boldsymbol{H}_{D}^{1+\kappa}(\Omega)\right)} \leq C_{1}\|l\|_{H^{1}\left(I ; \boldsymbol{L}^{2}(\Omega)\right)}, \\
&\left\|\varphi_{\tau}\right\|_{L^{\infty}\left(I ; H^{1+\theta}(\Omega)\right)} \leq C_{2}\left\{\left\|d_{\tau}\right\|_{L^{\infty}\left(I ; L^{2}(\Omega)\right)}+\|l\|_{H^{1}\left(I ; \boldsymbol{L}^{2}(\Omega)\right)}^{2}\right\},
\end{aligned}
$$

are fulfilled with constants $C_{1}=C_{1}\left(\left\|d_{\tau}\right\|_{L^{\infty}\left(I ; L^{2}(\Omega)\right)},\|l\|_{H^{1}\left(I ; L^{2}(\Omega)\right)}\right), C_{2}>0$ independent of $\tau$.

Proof. We only address higher spatial regularity since mere solvability has already been discussed. For $l \in$ $H^{1}\left(I ; \boldsymbol{L}^{2}(\Omega)\right)$, we have $P_{\tau} l_{\mid I_{m}} \in \boldsymbol{L}^{2}(\Omega)$ and

$$
\left\|P_{\tau} l_{\mid I_{m}}\right\|_{\boldsymbol{L}^{2}(\Omega)} \leq\left\|P_{\tau} l\right\|_{L^{\infty}\left(I ; \boldsymbol{L}^{2}(\Omega)\right)} \leq C\|l\|_{L^{\infty}\left(I ; \boldsymbol{L}^{2}(\Omega)\right)} \leq C\|l\|_{H^{1}\left(I ; \boldsymbol{L}^{2}(\Omega)\right)}
$$


for all $m=1, \ldots, M$. Moreover, we have $d_{\tau, m} \in H^{1}(\Omega)$ due to $\varphi_{\tau, m} \in H^{1}(\Omega)$ and Theorem 3.12 of [19]. Thus, Lemma 2.12 implies that $\boldsymbol{u}_{\tau, m} \in \boldsymbol{H}_{D}^{1+\kappa}(\Omega)$ for a $\kappa \in(0,1)$ and Lemma 2.14 then yields that $\varphi_{\tau, m} \in W^{2, \frac{p}{2}}(\Omega) \hookrightarrow$ $H^{1+\theta}(\Omega)$ with $\theta=\min \left\{1,2-\frac{4}{p}\right\}$. A second application of Theorem 3.12 from [19] yields $d_{\tau, m} \in H^{s}(\Omega)$ with $s=\min \left\{1+\theta, \frac{3}{2}-\epsilon\right\}$. Next, due to (2.21), we obtain

$$
\left\|\varphi_{\tau, m}\right\|_{H^{1+\theta}(\Omega)} \leq C_{2}\left\{\left\|d_{\tau, m}\right\|_{L^{2}(\Omega)}+\|l\|_{H^{1}\left(I ; \mathbf{L}^{2}(\Omega)\right)}^{2}\right\} .
$$

Since $C_{2}$ is independent of a particular subinterval $I_{m}$ we arrive at (3.10). Next, (2.19) and $\boldsymbol{L}^{2}(\Omega) \hookrightarrow \boldsymbol{H}^{\kappa-1}(\Omega)$ yield

$$
\left\|\boldsymbol{u}_{\tau, m}\right\|_{\boldsymbol{H}_{D}^{1+\kappa}(\Omega)} \leq C_{1}\left\|P_{\tau} l_{\mid I_{m}}\right\|_{\boldsymbol{L}^{2}(\Omega)} \leq C_{1}\|l\|_{H^{1}\left(I ; \boldsymbol{L}^{2}(\Omega)\right)}
$$

Note, that according to Lemma 2.12, the last constant $C_{1}$ depends on the multiplier norm of $\varphi_{\tau, m}$. The just derived estimate (3.10) then yields the desired result.

Stability estimates for $d_{\tau}$ in $L^{\infty}\left(I ; H^{s}(\Omega)\right)$ require a preliminary result. We will thus present a stability estimate for $d_{\tau}$ in $L^{\infty}\left(I ; L^{2}(\Omega)\right)$ first:

Lemma 3.2. Let $d_{\tau} \in L^{\infty}\left(I ; L^{2}(\Omega)\right)$ be the solution of (3.8) for a given initial datum $d_{0} \in L^{2}(\Omega)$ and a load $l \in L^{\infty}\left(I ; \boldsymbol{W}^{-1, p}(\Omega)\right)$. Then, we have

$$
\left\|d_{\tau}\right\|_{L^{\infty}\left(I ; L^{2}(\Omega)\right)} \leq C\left\{\|l\|_{L^{1}\left(I ; \boldsymbol{W}^{-1, p}(\Omega)\right)}+\left\|d_{0}\right\|\right\}
$$

with a constant $C>0$ independent of $\tau$ provided that $\tau$ is sufficiently small.

Proof. The lemma can be proven along the lines of the proof of Lemma 3.2 of [19].

Lemma 3.3. Let $d_{\tau} \in L^{\infty}\left(I ; H^{s}(\Omega)\right)$ be the solution of (3.8) for a given initial datum $d_{0} \in H^{s}(\Omega), s \in\left[1, \frac{3}{2}\right)$, and a load $l \in H^{1}\left(I ; \boldsymbol{L}^{2}(\Omega)\right)$. Then, we have

$$
\left\|d_{\tau}\right\|_{L^{\infty}\left(I ; H^{s}(\Omega)\right)} \leq C\left\{\|l\|_{H^{1}\left(I ; \boldsymbol{L}^{2}(\Omega)\right)}+\|l\|_{H^{1}\left(I ; \mathbf{L}^{2}(\Omega)\right)}^{2}+\left\|d_{0}\right\|_{H^{s}(\Omega)}\right\}
$$

with a constant $C>0$ independent of $\tau$ provided that $\tau$ is sufficiently small.

Proof. The lemma can be proven along the lines of the proof of Lemma 3.14 of [19], since Lemma 3.2 and Theorem 3.1 imply that

$$
\left\|\varphi_{\tau}\right\|_{L^{\infty}\left(I ; H^{s}(\Omega)\right)} \leq C\left\{\left\|d_{0}\right\|+\|l\|_{H^{1}\left(I ; \mathbf{L}^{2}(\Omega)\right)}^{2}+\|l\|_{H^{1}\left(I ; \boldsymbol{L}^{2}(\Omega)\right)}\right\} .
$$

Remark 3.4. Note, that Lemma 3.2 ensures that the constant $C_{1}$ from Theorem 3.1 is only dependent of $\|l\|_{H^{1}\left(I ; \boldsymbol{L}^{2}(\Omega)\right)}$.

We turn our attention to the derivation of temporal discretization error estimates. Due to the special definition of the semidiscrete solutions $\boldsymbol{u}_{\tau}$ and $\varphi_{\tau}$, preliminary estimates for temporal errors are easily obtained from Lipschitz continuity of the solution operators $\mathcal{U}$ and $\Phi$. Indeed, we have

$$
\left\|\boldsymbol{u}(t)-\boldsymbol{u}_{\tau}(t)\right\|_{\boldsymbol{W}_{D}^{1,2}(\Omega)} \leq L_{\mathcal{U}}\left\{\left\|l(t)-P_{\tau} l(t)\right\|_{\boldsymbol{W}^{-1, p}(\Omega)}+\left\|\varphi(t)-\varphi_{\tau}(t)\right\|_{H^{1}(\Omega)}\right\}
$$

and

$$
\left\|\varphi(t)-\varphi_{\tau}(t)\right\|_{H^{1}(\Omega)} \leq L_{\Phi}\left\{\left\|l(t)-P_{\tau} l(t)\right\|_{W^{-1, p}(\Omega)}+\left\|d(t)-d_{\tau}(t)\right\|_{L^{2}(\Omega)}\right\} .
$$

Thus, a projection error estimate for $l$ and an error estimate for the temporal error of $d-d_{\tau}$ directly lead to error estimates for $\boldsymbol{u}-\boldsymbol{u}_{\tau}$ and $\varphi-\varphi_{\tau}$. We still have to decide which norm we want to choose for the temporal 
error estimation. The possible norms for $\boldsymbol{u}$ and $\varphi$ depend on the projection error estimates available for $l$. Since we assume $l \in H^{1}\left(I ; \boldsymbol{L}^{2}(\Omega)\right)$ we choose $L^{2}(I ; \cdot)$-norms for $\boldsymbol{u}$ and $\varphi$. But for $d$, it is possible to derive $L^{\infty}\left(I ; L^{2}(\Omega)\right)$-error estimates. In Ch. 2 of [32], Susu proves linear convergence of the implicit Euler method applied to the reduced ODE in $L^{\infty}\left(I ; L^{2}(\Omega)\right)$. It is well known that the implicit Euler method follows from the piecewise constant discontinuous Galerkin method by approximating the right-hand side $P_{\tau} l_{I_{m}}$ by $l\left(t_{m}\right)$. Therefore, both methods are highly linked and we want to establish error estimates in $L^{\infty}\left(I ; L^{2}(\Omega)\right)$ as well. Note, that our proof differs from the method employed in [32] as we work with a semidiscrete solution which is discontinuous in time whereas Susu assumes continuity in time for the semidiscrete solution. Convergence of the implicit Euler method in $L^{\infty}\left(I ; L^{2}(\Omega)\right)$ is a byproduct from the convergence in $W^{1, \infty}\left(I ; L^{2}(\Omega)\right)$ which is proven in [32] whereas we will prove convergence directly. Note further, that Susu also provides an error estimate for $\varphi-\varphi_{\tau}$ in the $L^{\infty}\left(I ; L^{2}(\Omega)\right)$-norm. This is possible as she assumes Lipschitz continuity with respect to time for the control $l$, i.e. such a result requires $l \in W^{1, \infty}\left(I ; \boldsymbol{W}^{-1, p}(\Omega)\right)$.

We establish an error estimate for $d-d_{\tau}$ in two steps working only with the ODE. Recall, that the continuous ODE was given as

$$
\partial_{t} d(t)=\frac{1}{\delta} \max (-\beta(d(t)-\varphi(t))-r), \quad d(0)=d_{0}
$$

for almost all $t \in[0, T]$ with $\varphi(t)=\Phi(l(t), d(t))$ while the semidiscrete ODE is given as

$$
d_{\tau, m}=d_{\tau, m-1}+\frac{\tau_{m}}{\delta} \max \left(-\beta\left(d_{\tau, m}-\varphi_{\tau, m}\right)-r\right), \quad d_{\tau}(0)=d_{0}
$$

for all $m=1, \ldots, M$ with $\varphi_{\tau, m}=\Phi\left(P_{\tau} l_{\left.\right|_{m}}, d_{\tau, m}\right)$.

We will split the error $d-d_{\tau}=d-\tilde{d}+\tilde{d}-d_{\tau}$ where $\tilde{d} \in W^{1, \infty}\left(I ; L^{2}(\Omega)\right)$ is the unique solution of the auxiliary problem

$$
\partial_{t} \tilde{d}(t)=\frac{1}{\delta} \max \left(-\beta\left(\tilde{d}(t)-\varphi_{\tau}(t)\right)-r\right), \quad \tilde{d}(0)=d_{0}
$$

for almost all $t \in[0, T]$ with $\varphi_{\tau}(t)=\Phi\left(P_{\tau} l(t), d_{\tau}(t)\right)$. The existence of a unique solution $\tilde{d} \in W^{1, \infty}\left(I ; L^{2}(\Omega)\right)$ can be proven by means of Picard-Lindelöf's theorem in abstract function spaces (cf. [8]) as the right-hand side is Lipschitz continuous w.r.t. $\tilde{d}$. We start with the error between the solutions of (3.15) and (3.17).

Lemma 3.5. Let $d \in W^{1, \infty}\left(I ; L^{2}(\Omega)\right)$ be the solution of $(3.15)$ and $\tilde{d} \in W^{1, \infty}\left(I ; L^{2}(\Omega)\right)$ be the solution of (3.17) for a given load $l \in L^{\infty}\left(I ; \boldsymbol{W}^{-1, p}(\Omega)\right)$ and an initial state $d_{0} \in L^{2}(\Omega)$. Then, we have the a priori error estimate

$$
\|d-\tilde{d}\|_{L^{\infty}\left(I ; L^{2}(\Omega)\right)} \leq C\left\{\left\|l-P_{\tau} l\right\|_{L^{1}\left(I ; \boldsymbol{W}^{-1, p}(\Omega)\right)}+\left\|\tilde{d}-d_{\tau}\right\|_{L^{1}\left(I ; L^{2}(\Omega)\right)}\right\}
$$

with a constant $C>0$ independent of $\tau$.

Proof. The lemma can by proven by employing Gronwall's inequality. We rewrite $d$ and $\tilde{d}$ as

$$
\begin{aligned}
& d(t)=d_{0}+\frac{1}{\delta} \int_{0}^{t} \max (-\beta(d(s)-\varphi(s))-r) \mathrm{ds} \\
& \tilde{d}(t)=d_{0}+\frac{1}{\delta} \int_{0}^{t} \max \left(-\beta\left(\tilde{d}(s)-\varphi_{\tau}(s)\right)-r\right) \mathrm{ds}
\end{aligned}
$$

for almost all $t \in[0, T]$. Due to the Lipschitz continuity of max and $\Phi$ and Lem. 2.2, we obtain

$$
\begin{aligned}
\|d(t)-\tilde{d}(t)\| & \leq \frac{1}{\delta} \int_{0}^{t} \beta\left\{\|d(s)-\tilde{d}(s)\|+\left\|\varphi(s)-\varphi_{\tau}(s)\right\|\right\} \mathrm{ds} \\
& \leq \underbrace{\frac{\beta}{\delta}\left(1+L_{\Phi}\right)}_{\gamma(t)} \int_{0}^{t}\|d(s)-\tilde{d}(s)\| \mathrm{ds}+\underbrace{\frac{\beta}{\delta} L_{\Phi} \int_{0}^{t}\left\|l(s)-P_{\tau} l(s)\right\|_{W^{-1, p}(\Omega)}+\left\|\tilde{d}(s)-d_{\tau}(s)\right\| \mathrm{ds}}_{\alpha(t)} .
\end{aligned}
$$


As $\gamma(t)$ is constant and $\alpha(t)$ is monotone increasing, Gronwall's inequality yields the desired result.

The second part of the error will also be proven with Gronwall's inequality. But, as $\tilde{d}-d_{\tau}$ is continuous in time only on each subinterval $I_{m}$, we have to split the overall time horizon $I$ into the subintervals.

Lemma 3.6. Let $\tilde{d} \in W^{1, \infty}\left(I ; L^{2}(\Omega)\right)$ be the solution of $(3.17)$ and let $d_{\tau} \in L^{\infty}\left(I ; L^{2}(\Omega)\right)$ be the solution of (3.16) for a given load $l \in L^{\infty}\left(I ; \boldsymbol{W}^{-1, p}(\Omega)\right)$ and an initial state $d_{0} \in L^{2}(\Omega)$. Then, we have the error estimate

$$
\left\|\tilde{d}-d_{\tau}\right\|_{L^{\infty}\left(I ; L^{2}(\Omega)\right)} \leq C \tau
$$

with a constant $C>0$ independent of $\tau$.

Proof. At first, we have

$$
\frac{\beta}{\delta}\left\|d_{\tau}-\varphi_{\tau}\right\|_{L^{\infty}\left(I ; L^{2}(\Omega)\right)} \leq \tilde{C}
$$

according to Theorem 3.1 and Lemma 3.2 with a constant $\tilde{C}>0$ depending (among others) on $l$ and $d_{0}$, but not on $\tau$. We will need this estimate later. Next for $t \in I_{k}, k=1, \ldots, M$, we rewrite (3.17) as

$$
\tilde{d}(t)=d_{0}+\sum_{m=1}^{k-1} \frac{1}{\delta} \int_{I_{m}} \max \left(-\beta\left(\tilde{d}(s)-\varphi_{\tau, m}\right)-r\right) \mathrm{d} s+\frac{1}{\delta} \int_{t_{k-1}}^{t} \max \left(-\beta\left(\tilde{d}(s)-\varphi_{\tau, k}\right)-r\right) \mathrm{d} s
$$

and (3.16) as

$$
d_{\tau}(t)=d_{\tau, k}=d_{0}+\sum_{m=1}^{k} \frac{1}{\delta} \int_{I_{m}} \max \left(-\beta\left(d_{\tau, m}-\varphi_{\tau, m}\right)-r\right) \mathrm{ds} .
$$

Then, we obtain for $t \in I_{k}$

$$
\begin{aligned}
\left\|\tilde{d}(t)-d_{\tau}(t)\right\| & \leq \frac{\beta}{\delta} \sum_{m=1}^{k-1} \int_{I_{m}}\left\|\tilde{d}(s)-d_{\tau}(s)\right\| \mathrm{d} \mathrm{s}+\frac{\beta}{\delta} \int_{t_{k-1}}^{t}\left\|\tilde{d}(s)-d_{\tau}(s)\right\| \mathrm{d} \mathrm{s}+\frac{\beta}{\delta} \int_{t}^{t_{k}}\left\|d_{\tau, k}-\varphi_{\tau, k}\right\| \mathrm{ds} \\
& \leq \frac{\beta}{\delta} \int_{0}^{t}\left\|\tilde{d}(s)-d_{\tau}(s)\right\| \mathrm{ds}+\tilde{C} \tau .
\end{aligned}
$$

Gronwall's lemma then yields

$$
\left\|\tilde{d}(t)-d_{\tau}(t)\right\| \leq \tilde{C} \tau \exp \left(\frac{\beta}{\delta} T\right)
$$

The assertion now follows as the right-hand side is independent of $t \in I_{k}$.

Finally, we are able to state an a priori error estimate for $d-d_{\tau}$.

Theorem 3.7. For the error between the continuous solution $d \in W^{1, \infty}\left(I ; L^{2}(\Omega)\right)$ and the semidiscrete solution $d_{\tau} \in X_{\tau}^{0}$ for a given load $l \in H^{1}\left(I ; \boldsymbol{L}^{2}(\Omega)\right)$ and a given initial state $d_{0} \in L^{2}(\Omega)$, we have the error estimate

$$
\left\|d-d_{\tau}\right\|_{L^{\infty}\left(I ; L^{2}(\Omega)\right)} \leq C \tau
$$

with a constant $C>0$ independent of $\tau$.

Proof. We just need to combine the results of Lemmas 3.5 and 3.6. This gives us

$$
\left\|d-d_{\tau}\right\|_{L^{\infty}\left(I ; L^{2}(\Omega)\right)} \leq C\left\{\left\|l-P_{\tau} l\right\|_{L^{1}\left(I ; \boldsymbol{W}^{-1, p}(\Omega)\right)}+\left\|\tilde{d}-d_{\tau}\right\|_{L^{1}\left(I ; L^{2}(\Omega)\right)}+\tau\right\} .
$$

The error estimate for $\tilde{d}-d_{\tau}$ in the $L^{\infty}\left(I ; L^{2}(\Omega)\right)$-norm is also an error estimate for the $L^{1}\left(I ; L^{2}(\Omega)\right)$ norm and therefore this term also converges linear. Due to $l \in H^{1}\left(I ; \boldsymbol{L}^{2}(\Omega)\right)$ we have

$$
\left\|l-P_{\tau} l\right\|_{L^{1}\left(I ; \boldsymbol{W}^{-1, p}(\Omega)\right)} \leq C \tau \text {. }
$$

Thus, we arrive at the desired result. 
Based on (3.13) and (3.14), we arrive at the following overall temporal error estimate

Theorem 3.8. For a given load $l \in H^{1}\left(I ; \boldsymbol{L}^{2}(\Omega)\right)$ and an initial state $d_{0} \in L^{2}(\Omega)$, let $(\boldsymbol{u}, \varphi, d) \in$ $L^{\infty}\left(I ; \boldsymbol{W}_{D}^{1,2}(\Omega)\right) \times L^{\infty}\left(I ; H^{1}(\Omega)\right) \times W^{1, \infty}\left(I ; L^{2}(\Omega)\right)$ be the solution of the continuous damage model and let $\left(\boldsymbol{u}_{\tau}, \varphi_{\tau}, d_{\tau}\right) \in \boldsymbol{U}_{\tau}^{0}(p) \times V_{\tau}^{0} \times X_{\tau}^{0}$ be the solution of the semidiscrete damage model. Then, we have the error estimate

$$
\left\|\boldsymbol{u}-\boldsymbol{u}_{\tau}\right\|_{L^{2}\left(I ; \boldsymbol{W}_{D}^{1,2}(\Omega)\right)}+\left\|\varphi-\varphi_{\tau}\right\|_{L^{2}\left(I ; H^{1}(\Omega)\right)}+\left\|d-d_{\tau}\right\|_{L^{\infty}\left(I ; L^{2}(\Omega)\right)} \leq C \tau
$$

with a constant $C>0$ independent of $\tau$.

\section{DiscRetization in SPACE}

In this section, we provide error estimates for the spatial discretization.

\subsection{Discretization in space}

We begin with introducing the latter. We use $H^{1}$-conforming linear finite elements in space. Thus, we consider a quasi-uniform mesh $\mathbb{T}_{h}$ of shape regular triangles $\mathcal{T}$, which do not overlap and cover the domain $\Omega$. By $h_{\mathcal{T}}$ we denote the diameter of the triangle $\mathcal{T}$ and $h$ is the maximal triangle diameter. On the mesh $\mathbb{T}_{h}$ we construct two conforming finite element spaces

$$
\begin{aligned}
\boldsymbol{U}_{h}^{1} & =\left\{\boldsymbol{u} \in C\left(\bar{\Omega} ; \mathbb{R}^{2}\right): \boldsymbol{u}_{\mid \mathcal{T}} \in \mathbb{P}_{1}\left(\mathcal{T} ; \mathbb{R}^{2}\right), \mathcal{T} \in \mathbb{T}_{h}, u_{\mid \Gamma_{D}}=0\right\} \\
X_{h}^{1} & =\left\{v \in C(\bar{\Omega}): v_{\mid \mathcal{T}} \in \mathbb{P}_{1}(\mathcal{T}), \mathcal{T} \in \mathbb{T}_{h}\right\}
\end{aligned}
$$

Then the space-time discrete finite element spaces are given by

$$
\begin{aligned}
& \boldsymbol{U}_{\tau h}^{0,1}=\left\{u \in L^{2}\left(I, \boldsymbol{U}_{h}^{1}\right): \boldsymbol{u}_{\mid I_{m}} \in \mathbb{P}_{0}\left(I_{m} ; \boldsymbol{U}_{h}^{1}\right)\right\} \subset \boldsymbol{U}_{\tau}^{0}(p) \subset \boldsymbol{U}_{\tau}^{0}\left(p^{\prime}\right), \\
& X_{\tau h}^{0,1}=\left\{v \in L^{2}\left(I, V_{h}^{1}\right): v_{\mid I_{m}} \in \mathbb{P}_{0}\left(I_{m} ; X_{h}^{1}\right)\right\} \subset V_{\tau}^{0} \subset X_{\tau}^{0} .
\end{aligned}
$$

The space-time discrete equations then read as follows: Find states $\left(\boldsymbol{u}_{\tau h}, \varphi_{\tau h}, d_{\tau h}\right) \in \boldsymbol{U}_{\tau h}^{0,1} \times X_{\tau h}^{0,1} \times X_{\tau h}^{0,1}$ such that

$$
\begin{aligned}
\left(g\left(\varphi_{\tau h}\right) \mathbb{C} \varepsilon\left(\boldsymbol{u}_{\tau h}\right), \varepsilon(v)\right)_{I \times \Omega}= & \langle l, v\rangle_{I \times \Omega} \\
& -\alpha\left(\nabla \varphi_{\tau h}, \nabla \psi\right)_{I \times \Omega}+\beta\left(\varphi_{\tau h}, \psi\right)_{I \times \Omega} \\
+\frac{1}{2}\left(g^{\prime}\left(\varphi_{\tau h}\right) \mathbb{C} \varepsilon\left(\boldsymbol{u}_{\tau h}\right): \varepsilon\left(\boldsymbol{u}_{\tau h}\right), \psi\right)_{I \times \Omega}= & \beta\left(d_{\tau h}, \psi\right)_{I \times \Omega} \\
& \sum_{m=1}^{M}\left(\partial_{t} d_{\tau h}, \lambda\right)_{I_{m} \times \Omega}+\sum_{m=2}^{M}\left(\left[d_{\tau h}\right]_{m-1}, \lambda_{m-1}^{+}\right) \\
+\left(d_{\tau h, 0}^{+}, \lambda_{0}^{+}\right)= & \frac{1}{\delta}\left(\max \left(-\beta\left(d_{\tau h}-\varphi_{\tau h}\right)-r\right), \lambda\right)_{I \times \Omega}+\left(d_{0}, \lambda_{0}^{+}\right)
\end{aligned}
$$

is fulfilled for all $(v, \psi, \lambda) \in \boldsymbol{U}_{\tau h}^{0,1} \times X_{\tau h}^{0,1} \times X_{\tau h}^{0,1}$.

For the projection onto $\boldsymbol{U}_{\tau h}^{0,1}$ and $X_{\tau h}^{0,1}$ we work with the standard $L^{2}$-projection $\boldsymbol{P}_{h}^{U}: \boldsymbol{L}^{2}(\Omega) \rightarrow \boldsymbol{U}_{h}^{1}, P_{h}^{X}:$ $L^{2}(\Omega) \rightarrow X_{h}^{1}$ in space on each subinterval $I_{m}$ and define the time-space projection $\pi_{h}^{U}: \boldsymbol{U}_{\tau}^{0} \rightarrow \boldsymbol{U}_{\tau h}^{0,1}, \pi_{h}^{X}: X_{\tau}^{0} \rightarrow$ $X_{\tau h}^{0,1}$ via $\left(\pi_{h} z\right)(t)=P_{h}(z(t))$. 


\subsection{Spatial error estimates}

In contrast to temporal error estimates, spatial error estimates are not derived that easily. We have to derive spatial error estimates in several steps. At first, we establish preliminary error estimates for $\boldsymbol{u}_{\tau}-\boldsymbol{u}_{\tau h}$ and $\varphi_{\tau}-\varphi_{\tau h}$ in $H^{1}(\Omega)$ and $L^{2}(\Omega)$ on each subinterval $I_{m}$. In the next step, we show an error estimate for $d_{\tau}-d_{\tau h}$ in $L^{2}\left(I ; L^{2}(\Omega)\right)$ which leads to error estimates for $\boldsymbol{u}_{\tau}-\boldsymbol{u}_{\tau h}$ and $\varphi_{\tau}-\varphi_{\tau h}$ in $L^{2}\left(I ; H^{1}(\Omega)\right)$ and $L^{2}\left(I ; L^{2}(\Omega)\right)$ as well.

Existence of a unique solution of the system (4.1)-(4.3) will be discussed throughout the whole section. Note, that it suffices to prove existence of a unique solution on each subinterval $I_{m}$ in order to show unique solvability of (4.1)-(4.3).

$H^{1}(\Omega)$-error estimate for $\boldsymbol{u}_{\tau}-\boldsymbol{u}_{\tau h}$

We start with an error estimate for $u_{\tau, m}-u_{\tau h, m}$ in $\boldsymbol{W}_{D}^{1,2}(\Omega)$ on each subinterval $I_{m}$. To this end, we consider (3.1) and (4.1) on one single subinterval $I_{m}$. The corresponding equations are given as

$$
\left(g\left(\varphi_{\tau, m}\right) \mathbb{C} \varepsilon\left(\boldsymbol{u}_{\tau, m}\right), \varepsilon(v)\right)=\left\langle P_{\tau} l_{I_{m}}, v\right\rangle \quad \forall v \in \boldsymbol{W}_{D}^{1, p^{\prime}}(\Omega)
$$

and

$$
\left(g\left(\varphi_{\tau h, m}\right) \mathbb{C} \varepsilon\left(\boldsymbol{u}_{\tau h, m}\right), \varepsilon(v)\right)=\left\langle P_{\tau} l_{\left.\right|_{I m}}, v\right\rangle \quad \forall v \in \boldsymbol{U}_{h}^{1} .
$$

At first, we address unique solvability of (4.1) or equivalently unique solvability of (4.5) for a given $\varphi_{\tau h} \in X_{h}^{1}$ and $l \in \boldsymbol{W}^{-1, p}(\Omega)$.

Lemma 4.1. Let $\varphi \in L^{1}(\Omega)$ and $l \in \boldsymbol{W}^{-1, p}(\Omega)$ be given. Then, the equation

$$
\left\langle A_{\varphi} \boldsymbol{u}_{\tau h}, v\right\rangle:=\left(g(\varphi) \mathbb{C} \varepsilon\left(\boldsymbol{u}_{\tau h}\right), \varepsilon(v)\right)=\langle l, v\rangle \quad \forall v \in \boldsymbol{U}_{h}^{1}
$$

possesses a unique solution $\boldsymbol{u}_{\tau h} \in \boldsymbol{U}_{h}^{1}$.

Proof. Existence of a unique solution follows from the lemma of Lax-Milgram since the bilinear form $A_{\varphi}$ is bounded and coercive and $\boldsymbol{U}_{h}^{1}$ is a closed subspace of the Hilbert space $\boldsymbol{W}_{D}^{1,2}(\Omega)$.

We denote the solution operator of the above variational problem as $\mathcal{U}_{h}: \boldsymbol{W}^{-1, p}(\Omega) \times L^{1}(\Omega) \rightarrow \boldsymbol{U}_{h}^{1}, \mathcal{U}_{h}(l, \varphi)=$ $\boldsymbol{u}_{\tau h}$. As its continuous counterpart $\mathcal{U}$, the operator is Lipschitz continuous.

For the error estimation, we again split the error as $\boldsymbol{u}_{\tau, m}-\boldsymbol{u}_{\tau h, m}=\boldsymbol{u}_{\tau, m}-\tilde{\boldsymbol{u}}_{\tau h, m}+\tilde{\boldsymbol{u}}_{\tau h, m}-\boldsymbol{u}_{\tau h, m}$ where $\tilde{\boldsymbol{u}}_{\tau h, m}=\mathcal{U}_{h}\left(P_{\tau} l_{\left.\right|_{I m}}, \varphi_{\tau, m}\right) \in \boldsymbol{U}_{h}^{1}$ is the unique solution of the auxiliary problem

$$
\left(g\left(\varphi_{\tau, m}\right) \mathbb{C} \varepsilon\left(\tilde{\boldsymbol{u}}_{\tau h, m}\right), \varepsilon(v)\right)=\left\langle P_{\tau} l_{\left.\right|_{I_{m}}}, v\right\rangle \quad \forall v \in \boldsymbol{U}_{h}^{1} .
$$

An error estimate for $\tilde{\boldsymbol{u}}_{\tau h, m}-\boldsymbol{u}_{\tau h, m}$ follows directly from the Lipschitz continuity of $\mathcal{U}_{h}$.

Lemma 4.2. Let $\boldsymbol{u}_{\tau h, m}=\mathcal{U}_{h}\left(P_{\tau} l_{\left.\right|_{I m}}, \varphi_{\tau h, m}\right) \in \boldsymbol{U}_{h}^{1}$ and $\tilde{\boldsymbol{u}}_{\tau h, m}=\mathcal{U}_{h}\left(P_{\tau} l_{\left.\right|_{I_{m}}}, \varphi_{\tau, m}\right) \in \boldsymbol{U}_{h}^{1}$ be the solutions of (4.5) and (4.6) for a given load $l \in H^{1}\left(I ; \boldsymbol{L}^{2}(\Omega)\right)$ and given $\varphi_{\tau, m} \in H^{1}(\Omega), \varphi_{\tau h, m} \in X_{h}^{1}$. Then, we have the error estimate

$$
\left\|\tilde{\boldsymbol{u}}_{\tau h, m}-\boldsymbol{u}_{\tau h, m}\right\|_{\boldsymbol{W}_{D}^{1,2}(\Omega)} \leq C\left\|\varphi_{\tau, m}-\varphi_{\tau h, m}\right\|_{L^{\frac{2 p}{p-2}}(\Omega)}
$$

with a constant $C>0$ independent of $\tau, h$ and the subinterval $I_{m}$ for each $m=1, \ldots, M$.

An error estimate for $\boldsymbol{u}_{\tau, m}-\tilde{\boldsymbol{u}}_{\tau h, m}$ can now be established in a standard way by making use of the boundedness and coercivity of the bilinear form $A_{\varphi_{\tau, m}}: \boldsymbol{W}_{D}^{1,2}(\Omega) \rightarrow \boldsymbol{W}_{D}^{-1,2}(\Omega)$ for a given $\varphi_{\tau, m}$ :

Lemma 4.3. Let $\boldsymbol{u}_{\tau, m}=\mathcal{U}\left(P_{\tau} l_{\left.\right|_{I_{m}}}, \varphi_{\tau, m}\right) \in \boldsymbol{W}_{D}^{1, p}(\Omega)$ be the solution of (4.4) and let $\tilde{\boldsymbol{u}}_{\tau h, m}=\mathcal{U}_{h}\left(P_{\tau} l_{\left.\right|_{I m}}, \varphi_{\tau, m}\right) \in$ $\boldsymbol{U}_{h}^{1}$ be the solution of (4.6) for a given load $l \in H^{1}\left(I ; \boldsymbol{L}^{2}(\Omega)\right)$ and given $\varphi_{\tau, m} \in H^{1}(\Omega)$. Then, we have the error estimate

$$
\left\|\boldsymbol{u}_{\tau, m}-\tilde{\boldsymbol{u}}_{\tau h, m}\right\|_{\boldsymbol{W}_{D}^{1,2}(\Omega)} \leq C\left\|\boldsymbol{u}_{\tau, m}-\boldsymbol{P}_{h}^{U} \boldsymbol{u}_{\tau, m}\right\|_{W_{D}^{1,2}(\Omega)} \leq C h^{\kappa}\left\|\boldsymbol{u}_{\tau, m}\right\|_{\boldsymbol{H}_{D}^{1+\kappa}(\Omega)}
$$

with a constant $C>0$ independent of $\tau, h$ and the subinterval $I_{m}$ for each $m=1, \ldots, M$. 
Proof. As $\boldsymbol{U}_{h}^{1} \subset \boldsymbol{W}_{D}^{1, p^{\prime}}(\Omega)$, we have the property of Galerkin orthogonality, that is

$$
\left\langle A_{\varphi_{\tau, m}}\left(\boldsymbol{u}_{\tau, m}-\tilde{\boldsymbol{u}}_{\tau h, m}\right), v\right\rangle=0
$$

for all $v \in \boldsymbol{U}_{h}^{1}$. Thus, we obtain with the coercivity and boundedness of $A_{\varphi_{\tau, m}}$

$$
\left\|\boldsymbol{u}_{\tau, m}-\tilde{\boldsymbol{u}}_{\tau h, m}\right\|_{\boldsymbol{W}_{D}^{1,2}(\Omega)}^{2} \leq c\left\|\boldsymbol{u}_{\tau, m}-\tilde{\boldsymbol{u}}_{\tau h, m}\right\|_{\boldsymbol{W}_{D}^{1,2}(\Omega)}\left\|\boldsymbol{u}_{\tau, m}-\boldsymbol{P}_{h}^{U} \boldsymbol{u}_{\tau, m}\right\|_{W_{D}^{1,2}(\Omega)} .
$$

Then, the assertion follows directly from the error estimate for the projection $\boldsymbol{P}_{h}^{U}$. This error estimate for $\boldsymbol{u} \in \boldsymbol{H}_{D}^{1+\kappa}(\Omega)$ on $\boldsymbol{W}_{D}^{1,2}(\Omega)$ can be obtained as in the case $u \in H^{1+\kappa}(\Omega)$ by making use of appropriate interpolation spaces and a corresponding error for the usual interpolation operator, see for example section 4 and 14 of [2].

Thus, we have the overall error estimate

$$
\left\|\boldsymbol{u}_{\tau, m}-\boldsymbol{u}_{\tau h, m}\right\|_{\boldsymbol{W}_{D}^{1,2}(\Omega)} \leq C\left\{\left\|\varphi_{\tau, m}-\varphi_{\tau h, m}\right\|_{L^{\frac{2 p}{p-2}(\Omega)}}+h^{\kappa}\left\|\boldsymbol{u}_{\tau, m}\right\|_{\boldsymbol{H}_{D}^{1+\kappa}(\Omega)}\right\}
$$

with a constant $C>0$ independent of $\tau, h$ and the subinterval $I_{m}$.

Error estimates for $\varphi_{\tau}-\varphi_{\tau h}$

Next, we derive an error estimate for $\varphi_{\tau, m}-\varphi_{\tau h, m}$ in $H^{1}(\Omega)$ on each subinterval $I_{m}$. The approach is similar to the $H^{1}(\Omega)$-error for $\boldsymbol{u}_{\tau, m}-\boldsymbol{u}_{\tau h, m}$. We may express (3.2) and (4.2) with $B$ and $F$ (see Def. 2.5) as

$$
\left\langle B \varphi_{\tau, m}+F\left(P_{\tau} l_{\left.\right|_{m}}, \varphi_{\tau, m}\right), \psi_{m}\right\rangle=\beta\left(d_{\tau, m}, \psi_{m}\right) \quad \forall \psi_{m} \in H^{1}(\Omega)
$$

and

$$
\left\langle B \varphi_{\tau h, m}+F\left(P_{\tau} l_{\left.\right|_{I m}}, \varphi_{\tau h, m}\right), \psi_{m}\right\rangle=\beta\left(d_{\tau h, m}, \psi_{m}\right) \quad \forall \psi_{m} \in X_{h}^{1} .
$$

While unique solvability of (4.9) is clear, we still have to take a look at the unique solvability of (4.2). It suffices to prove the existence of a unique solution $\varphi_{\tau h, m}$ on each subinterval for a given right-hand side $d_{\tau h, m} \in L^{2}(\Omega)$.

Lemma 4.4. Let Assumption 2.1 be fulfilled. For given $l \in \boldsymbol{W}^{-1, p}(\Omega)$ and $d \in L^{2}(\Omega)$ the equation

$$
\left\langle B \varphi_{\tau h}+F\left(l, \varphi_{\tau h}\right), \psi_{h}\right\rangle=\beta\left(d, \psi_{h}\right) \quad \forall \psi_{h} \in X_{h}^{1}
$$

possesses a unique solution $\varphi_{\tau h} \in X_{h}^{1}$.

Proof. Existence of a solution may be proven with Brouwer's fixed point theorem. Uniqueness then follows due to the strong monotonicity of $B+F$ under Assumption 2.1.

We denote the discrete solution operator by $\Phi_{h}: \mathcal{B}_{\rho} \times L^{2}(\Omega) \rightarrow X_{h}^{1}, \Phi_{h}(l, d)=\varphi_{\tau h}$. Again, it is Lipschitz continuous as its continuous counterpart $\Phi$.

This time, we split the error as $\varphi_{\tau, m}-\varphi_{\tau h, m}=\varphi_{\tau, m}-\tilde{\varphi}_{\tau h, m}+\tilde{\varphi}_{\tau h, m}-\varphi_{\tau h, m}$ with $\tilde{\varphi}_{\tau h, m}=\Phi_{h}\left(P_{\tau} l_{\left.\right|_{I m}}, d_{\tau, m}\right) \in$ $X_{h}^{1}$ being the unique solution of the auxiliary equation

$$
\left\langle B \tilde{\varphi}_{\tau h, m}+F\left(P_{\tau} l_{\left.\right|_{I_{m}}}, \tilde{\varphi}_{\tau h, m}\right), \psi_{m}\right\rangle=\beta\left(d_{\tau, m}, \psi_{m}\right) \quad \forall \psi_{m} \in X_{h}^{1} .
$$

As in the previous paragraph, an error estimate for $\tilde{\varphi}_{\tau h, m}-\varphi_{\tau h, m}$ follows immediately from the Lipschitz continuity of $\Phi_{h}$ :

Lemma 4.5. Let $\tilde{\varphi}_{\tau h, m}=\Phi_{h}\left(P_{\tau} l_{\left.\right|_{I_{m}}}, d_{\tau, m}\right) \in X_{h}^{1}$ and $\varphi_{\tau h, m}=\Phi_{h}\left(P_{\tau} l_{\left.\right|_{I_{m}}}, d_{\tau h, m}\right) \in X_{h}^{1}$ be the solutions of (4.11) and (4.10) for a given right-hand side $l \in H^{1}\left(I ; \boldsymbol{L}^{2}(\Omega)\right) \cap \mathcal{B}_{\rho}^{\infty}$ and given $d_{\tau, m} \in L^{2}(\Omega), d_{\tau h, m} \in X_{h}^{1}$. Then, we have the error estimate

$$
\left\|\tilde{\varphi}_{\tau h, m}-\varphi_{\tau h, m}\right\|_{L^{r}(\Omega)} \leq\left\|\tilde{\varphi}_{\tau h, m}-\varphi_{\tau h, m}\right\|_{H^{1}(\Omega)} \leq L_{\Phi}\left\|d_{\tau, m}-d_{\tau h, m}\right\|_{L^{2}(\Omega)}
$$

for all $r \in[1, \infty)$. 
The error estimate for $\varphi_{\tau, m}-\tilde{\varphi}_{\tau h, m}$ will be proven with the strong monotonicity property of $B+F$ (see $(2.10))$.

Lemma 4.6. Let Assumption 2.1 hold true. For functions $l \in H^{1}\left(I ; L^{2}(\Omega)\right) \cap \mathcal{B}_{\rho}^{\infty}$ and $d_{\tau, m} \in L^{2}(\Omega)$, let the functions $\tilde{\varphi}_{\tau h, m}=\Phi_{h}\left(P_{\tau} l_{\left.\right|_{m}}, d_{\tau, m}\right) \in X_{h}^{1}$ and $\varphi_{\tau, m}=\Phi\left(P_{\tau} l_{\left.\right|_{I_{m}}}, d_{\tau, m}\right) \in H^{1}(\Omega)$ be the solutions of (4.11) and (4.9). Then, we have the error estimate

$$
\left\|\varphi_{\tau, m}-\tilde{\varphi}_{\tau h, m}\right\|_{H^{1}(\Omega)} \leq C h^{\theta}\left\|\varphi_{\tau, m}\right\|_{H^{1+\theta}(\Omega)}
$$

with a constant $C>0$ depending on $\rho$ but independent of $\tau, h$ and the subinterval $I_{m}$.

Proof. As $X_{h}^{1} \subset H^{1}(\Omega)$, we have the property of Galerkin orthogonality, that is

$$
\left\langle B\left(\varphi_{\tau, m}-\tilde{\varphi}_{\tau h, m}\right)+F\left(P_{\tau} l_{\left.\right|_{I m}}, \varphi_{\tau, m}\right)-F\left(P_{\tau} l_{\left.\right|_{I m}}, \tilde{\varphi}_{\tau h, m}\right), \psi_{m}\right\rangle=0
$$

for all $\psi_{m} \in X_{h}^{1}$. Then, we obtain with strong monotonicity of $B+F$, boundedness of $B$ and Lipschitz continuity of $F$

$$
\begin{aligned}
\frac{\alpha}{2}\left\|\varphi_{\tau, m}-\tilde{\varphi}_{\tau h, m}\right\|_{H^{1}(\Omega)}^{2} & =\left\langle B\left(\varphi_{\tau, m}-\tilde{\varphi}_{\tau h, m}\right)+F\left(P_{\tau} l_{\left.\right|_{I_{m}}}, \varphi_{\tau, m}\right)-F\left(P_{\tau} l_{\left.\right|_{I_{m}}}, \tilde{\varphi}_{\tau h, m}\right), \varphi_{\tau, m}-P_{h} \varphi_{\tau, m}\right\rangle \\
& \leq C(\rho)\left\|\varphi_{\tau, m}-\tilde{\varphi}_{\tau h, m}\right\|_{H^{1}(\Omega)}\left\|\varphi_{\tau, m}-P_{h} \varphi_{\tau, m}\right\|_{H^{1}(\Omega)} .
\end{aligned}
$$

Lemmas 4.6 and 4.5 combined then yield the overall error estimate

$$
\left\|\varphi_{\tau, m}-\varphi_{\tau h, m}\right\|_{H^{1}(\Omega)} \leq C(\rho) h^{\theta}\left\|\varphi_{\tau, m}\right\|_{H^{1+\theta}(\Omega)}+L_{\Phi}\left\|d_{\tau, m}-d_{\tau h, m}\right\|_{L^{2}(\Omega)} .
$$

We will also need an $L^{2}(\Omega)$-error estimate for $\varphi_{\tau, m}-\varphi_{\tau h, m}$. We already know from Lemma 4.5 that

$$
\left\|\tilde{\varphi}_{\tau h, m}-\varphi_{\tau h, m}\right\|_{L^{r}(\Omega)} \leq L_{\Phi}\left\|d_{\tau, m}-d_{\tau h, m}\right\|_{L^{2}(\Omega)}
$$

for all $r \geq 1$. Therefore, we only need an error estimate for $\varphi_{\tau, m}-\tilde{\varphi}_{\tau h, m}$. This will be established via the Aubin-Nitsche-Trick. We will provide a more general result, that is, we will derive an error estimate in $L^{r}(\Omega)$ with $r \geq 1$ arbitrary.

Let an arbitrary right-hand side $f \in H^{1}(\Omega) \cap L^{r^{\prime}}(\Omega)$ be given. Moreover, we assume that $l \in H^{1}\left(I ; \boldsymbol{L}^{2}(\Omega)\right) \cap$ $\mathcal{B}_{\rho}^{1,2}:=\left\{l \in H^{1}\left(I ; \boldsymbol{L}^{2}(\Omega)\right):\|l\|_{H^{1}\left(I ; \boldsymbol{L}^{2}(\Omega)\right)}<\rho\right\}$. We define the following dual problem: Find a state $z \in H^{1}(\Omega)$ such that

$$
\left\langle B \psi+\partial_{\varphi} F\left(P_{\tau} l_{\left.\right|_{I m}}, \varphi_{\tau, m}\right) \psi, z\right\rangle=(f, \psi)
$$

is fulfilled for all $\psi \in H^{1}(\Omega)$. Note, that $B$ as well as $\partial_{\varphi} F(l, \varphi)$ are symmetric, that is, the above problem is equivalent to

$$
\left\langle B z+\partial_{\varphi} F\left(P_{\tau} l_{I_{m}}, \varphi_{\tau, m}\right) z, \psi\right\rangle=(f, \psi) .
$$

Unique solvability of this equation is shown in Lemma 2.15. Therefore, there exists a unique $z \in H^{1}(\Omega) \cap W^{2, \omega}(\Omega)$ which fulfills

$$
\|z\|_{W^{2, \omega}(\Omega)} \leq C\|f\|_{L^{r^{\prime}(\Omega)}}
$$

with a constant $C>0$ depending only on $\rho$ for a given $l \in \mathcal{B}_{\rho}^{1,2}$ and with $\omega=\min \left\{r^{\prime}, \frac{p}{2}\right\}$.

We test the dual equation with $\psi=\varphi_{\tau, m}-\tilde{\varphi}_{\tau h, m} \in H^{1}(\Omega)$. This leads to

$$
\begin{aligned}
& \left(f, \varphi_{\tau, m}-\tilde{\varphi}_{\tau h, m}\right)=\left\langle B\left(\varphi_{\tau, m}-\tilde{\varphi}_{\tau h, m}\right)+\partial_{\varphi} F\left(P_{\tau} l_{\left.\right|_{I_{m}}}, \varphi_{\tau, m}\right)\left(\varphi_{\tau, m}-\tilde{\varphi}_{\tau h, m}\right), z\right\rangle \\
& =\left\langle B\left(\varphi_{\tau, m}-\tilde{\varphi}_{\tau h, m}\right)+\partial_{\varphi} F\left(P_{\tau} l_{I_{I m}}, \varphi_{\tau, m}\right)\left(\varphi_{\tau, m}-\tilde{\varphi}_{\tau h, m}\right), z-P_{h} z\right\rangle \\
& +\left\langle B\left(\varphi_{\tau, m}-\tilde{\varphi}_{\tau h, m}\right)+\partial_{\varphi} F\left(P_{\tau} l_{\left.\right|_{I_{m}}}, \varphi_{\tau, m}\right)\left(\varphi_{\tau, m}-\tilde{\varphi}_{\tau h, m}\right), P_{h} z\right\rangle \\
& =\left\langle B\left(\varphi_{\tau, m}-\tilde{\varphi}_{\tau h, m}\right)+\partial_{\varphi} F\left(P_{\tau} l_{I_{I m}}, \varphi_{\tau, m}\right)\left(\varphi_{\tau, m}-\tilde{\varphi}_{\tau h, m}\right), z-P_{h} z\right\rangle \\
& +\left\langle F\left(P_{\tau} l_{\left.\right|_{I_{m}}}, \tilde{\varphi}_{\tau h, m}\right)-F\left(P_{\tau} l_{\left.\right|_{I_{m}}}, \varphi_{\tau, m}\right)+\partial_{\varphi} F\left(P_{\tau} l_{\left.\right|_{I_{m}}}, \varphi_{\tau, m}\right)\left(\varphi_{\tau, m}-\tilde{\varphi}_{\tau h, m}\right), P_{h} z\right\rangle
\end{aligned}
$$


where the last step follows from the Galerkin orthogonality (4.14). The boundedness of $B+\partial_{\varphi} F\left(P_{\tau} l_{\left.\right|_{I m}}, \varphi_{\tau, m}\right)$ established for the lemma of Lax-Milgram then gives us

$$
\begin{aligned}
\left(f, \varphi_{\tau, m}-\tilde{\varphi}_{\tau h, m}\right) \leq & C\left\|\varphi_{\tau, m}-\tilde{\varphi}_{\tau h, m}\right\|_{H^{1}(\Omega)}\left\|z-P_{h} z\right\|_{H^{1}(\Omega)} \\
& +\left\langle F\left(P_{\tau} l_{\left.\right|_{I_{m}}}, \tilde{\varphi}_{\tau h, m}\right)-F\left(P_{\tau} l_{\left.\right|_{I_{m}}}, \varphi_{\tau, m}\right)-\partial_{\varphi} F\left(P_{\tau} l_{\left.\right|_{I_{m}}}, \varphi_{\tau, m}\right)\left(\tilde{\varphi}_{\tau h, m}-\varphi_{\tau, m}\right), P_{h} z\right\rangle .
\end{aligned}
$$

Finally, we apply estimate (2.17) to the second term to arrive at

$$
\begin{aligned}
\left(f, \varphi_{\tau, m}-\tilde{\varphi}_{\tau h, m}\right) \leq & C\left\|\varphi_{\tau, m}-\tilde{\varphi}_{\tau h, m}\right\|_{H^{1}(\Omega)}\left\|z-P_{h} z\right\|_{H^{1}(\Omega)} \\
& +C\left\|\varphi_{\tau, m}-\tilde{\varphi}_{\tau h, m}\right\|_{H^{1}(\Omega)}^{2}\|z\|_{H^{1}(\Omega)} .
\end{aligned}
$$

For the projection error $z-P_{h} z$, we have for $z \in W^{2, \omega}(\Omega) \hookrightarrow H^{1+\gamma}(\Omega)$ with $\gamma=\min \left\{1, \frac{2}{r}, 2-\frac{4}{p}\right\}$ the usual error estimate

$$
\left\|z-P_{h} z\right\|_{H^{1}(\Omega)} \leq C h^{\gamma}\|z\|_{W^{2, \omega}(\Omega)} \leq C h^{\gamma}\|f\|_{L^{r^{\prime}}(\Omega)} .
$$

Together with $\|z\|_{H^{1}(\Omega)} \leq C\|f\|_{L^{r^{\prime}}(\Omega)}$ and the error estimate for $\varphi_{\tau, m}-\tilde{\varphi}_{\tau h, m}$ in $H^{1}(\Omega)$, we arrive at

$$
\left(f, \varphi_{\tau, m}-\tilde{\varphi}_{\tau h, m}\right) \leq C h^{\min \{\theta+\gamma, 2 \theta\}}\left(\left\|\varphi_{\tau, m}\right\|_{H^{1+\theta}(\Omega)}+\left\|\varphi_{\tau, m}\right\|_{H^{1+\theta}(\Omega)}^{2}\right)\|f\|_{L^{r^{\prime}}(\Omega)} .
$$

The stability estimates (3.10) and (3.12) yield boundedness of $\left\|\varphi_{\tau, m}\right\|_{H^{1+\theta}(\Omega)}+\left\|\varphi_{\tau, m}\right\|_{H^{1+\theta}(\Omega)}^{2} \leq C$ for all $m=1, \ldots, M$ with $C>0$ depending on $\left\|d_{0}\right\|$ and $\rho$ for $l \in \mathcal{B}_{\rho}^{1,2}$ but independent of $\tau$ and $m$. Finally, since $\theta=\min \left\{1,2-\frac{4}{p}\right\}$ and thus $\theta+1, \theta+2-\frac{4}{p} \geq 2 \theta$, we obtain the following result:

Lemma 4.7. Let Assumption 2.1 be fulfilled. Let $\varphi_{\tau, m}=\Phi\left(P_{\tau} l_{I_{m}}, d_{\tau, m}\right) \in H^{1}(\Omega)$ and $\varphi_{\tau h, m}=$ $\Phi_{h}\left(P_{\tau} l_{I_{m}}, d_{\tau h, m}\right) \in X_{h}^{1}$ be the solutions of (4.9) and (4.10) for a given right-hand side $l \in H^{1}\left(I ; \boldsymbol{L}^{2}(\Omega)\right) \cap B_{\rho}^{1,2}$ and given $d_{\tau, m} \in L^{2}(\Omega), d_{\tau h, m} \in X_{h}^{1}$. Then, we have the error estimate

$$
\left\|\varphi_{\tau, m}-\varphi_{\tau h, m}\right\|_{L^{r}(\Omega)} \leq C h^{\min \left\{\theta+\frac{2}{r}, 2 \theta\right\}}+L_{\Phi}\left\|d_{\tau, m}-d_{\tau h, m}\right\|_{L^{2}(\Omega)}
$$

with a constant $C>0$ independent of $\tau, h$ and the subinterval $I_{m}$ and for all $r \geq 1$.

For the special case $r=2$, we obtain a convergence rate of $2 \theta$ since $\theta+1 \geq 2 \theta$.

Therefore, it is suitable to formulate the following

Corollary 4.8. Let Assumption 2.1 be fulfilled and let $\varphi_{\tau, m}=\Phi\left(P_{\tau} l_{\left.\right|_{I m}}, d_{\tau, m}\right) \in H^{1}(\Omega)$ and $\varphi_{\tau h, m}=$ $\Phi_{h}\left(P_{\tau} l_{I_{m}}, d_{\tau h, m}\right) \in X_{h}^{1}$ be the solutions of (4.9) and (4.10) for a given right-hand side $l \in H^{1}\left(I ; \boldsymbol{L}^{2}(\Omega)\right) \cap B_{\rho}^{1,2}$ and given $d_{\tau, m} \in L^{2}(\Omega), d_{\tau h, m} \in X_{h}^{1}$. Then, we have the error estimate

$$
\left\|\varphi_{\tau, m}-\varphi_{\tau h, m}\right\|_{L^{2}(\Omega)} \leq C h^{2 \theta}+L_{\Phi}\left\|d_{\tau, m}-d_{\tau h, m}\right\|_{L^{2}(\Omega)}
$$

with a constant $C>0$ independent of $\tau, h$ and the subinterval $I_{m}$.

$L^{2}(\Omega)$-error estimate for $\boldsymbol{u}_{\tau}-\boldsymbol{u}_{\tau h}$

We need an error estimate for $\boldsymbol{u}_{\tau, m}-\boldsymbol{u}_{\tau h, m}$ in $L^{2}(\Omega)$ as well. We have according to Lemma 4.2

$$
\left\|\boldsymbol{u}_{\tau, m}-\boldsymbol{u}_{\tau h, m}\right\|_{L^{2}(\Omega)} \leq\left\|\boldsymbol{u}_{\tau, m}-\tilde{\boldsymbol{u}}_{\tau h, m}\right\|_{L^{2}(\Omega)}+C\left\|\varphi_{\tau, m}-\varphi_{\tau h, m}\right\|_{L^{\frac{2 p}{p-2}}(\Omega)}
$$

For $r=\frac{2 p}{p-2}$ in Lemma 4.7, we obtain

$$
\left\|\boldsymbol{u}_{\tau, m}-\boldsymbol{u}_{\tau h, m}\right\|_{L^{2}(\Omega)} \leq\left\|\boldsymbol{u}_{\tau, m}-\tilde{\boldsymbol{u}}_{\tau h, m}\right\|_{L^{2}(\Omega)}+C\left(h^{\min \left\{2 \theta, \theta+1-\frac{2}{p}\right\}}+L_{\Phi}\left\|d_{\tau, m}-d_{\tau h, m}\right\|_{L^{2}(\Omega)}\right) .
$$


Now, if $p>4$, we obtain $\theta=1$ and thus arrive at $2 \theta=2>2-\frac{2}{p}=\theta+1-\frac{2}{p}>\frac{3}{2}$. If $2<p \leq 4$, we obtain with $\theta=2-\frac{4}{p}$ the relation $\theta+1-\frac{2}{p}=\frac{3}{2} \theta<2 \theta$. Thus, in both cases the minimum is attained for $\theta+1-\frac{2}{p}$ which yields

$$
\left\|\boldsymbol{u}_{\tau, m}-\boldsymbol{u}_{\tau h, m}\right\|_{L^{2}(\Omega)} \leq\left\|\boldsymbol{u}_{\tau, m}-\tilde{\boldsymbol{u}}_{\tau h, m}\right\|_{L^{2}(\Omega)}+C\left(h^{\theta+1-\frac{2}{p}}+L_{\Phi}\left\|d_{\tau, m}-d_{\tau h, m}\right\|_{L^{2}(\Omega)}\right) .
$$

An estimate for $\boldsymbol{u}_{\tau, m}-\tilde{\boldsymbol{u}}_{\tau h, m}$ will be derived with the Aubin-Nitsche-Trick. We define $z \in \boldsymbol{W}_{D}^{1,2}(\Omega)$ as the unique solution of

$$
\left\langle A_{\varphi_{\tau, m}} v, z\right\rangle=\left(\boldsymbol{u}_{\tau, m}-\tilde{\boldsymbol{u}}_{\tau h, m}, v\right) \quad \forall v \in \boldsymbol{W}_{D}^{1,2}(\Omega) .
$$

As $A_{\varphi}$ is self adjoint, this problem is equivalent to

$$
\left\langle A_{\varphi_{\tau, m}} z, v\right\rangle=\left(\boldsymbol{u}_{\tau, m}-\tilde{\boldsymbol{u}}_{\tau h, m}, v\right)
$$

where the existence of a unique solution $z \in \boldsymbol{W}_{D}^{1,2}(\Omega) \cap \boldsymbol{H}_{D}^{1+\kappa}(\Omega)$ is known. Moreover, (2.19) and the embedding $\boldsymbol{H}_{D}^{1-\kappa}(\Omega) \hookrightarrow L^{2}(\Omega)$ give us

$$
\|z\|_{\boldsymbol{W}_{D}^{1,2}(\Omega)} \leq\|z\|_{\boldsymbol{H}_{D}^{1+\kappa}(\Omega)} \leq C\left\|\boldsymbol{u}_{\tau, m}-\tilde{\boldsymbol{u}}_{\tau h, m}\right\|_{L^{2}(\Omega)}
$$

We test the dual equation with $v=\boldsymbol{u}_{\tau, m}-\tilde{\boldsymbol{u}}_{\tau h, m} \in \boldsymbol{W}_{D}^{1,2}(\Omega)$ to arrive at

$$
\begin{aligned}
\left\|\boldsymbol{u}_{\tau, m}-\tilde{\boldsymbol{u}}_{\tau h, m}\right\|_{L^{2}(\Omega)}^{2} & =\left\langle A_{\varphi_{\tau, m}}\left(\boldsymbol{u}_{\tau, m}-\tilde{\boldsymbol{u}}_{\tau h, m}\right), z\right\rangle \\
& =\left\langle A_{\varphi_{\tau, m}}\left(\boldsymbol{u}_{\tau, m}-\tilde{\boldsymbol{u}}_{\tau h, m}\right), z-P_{h} z\right\rangle \\
& \leq C\left\|\boldsymbol{u}_{\tau, m}-\tilde{\boldsymbol{u}}_{\tau h, m}\right\|_{\boldsymbol{W}_{D}^{1,2}(\Omega)}\left\|z-P_{h} z\right\|_{W_{D}^{1,2}(\Omega)} \\
& \leq C h^{2 \kappa}\left\|\boldsymbol{u}_{\tau, m}\right\|_{\boldsymbol{H}_{D}^{1+\kappa}(\Omega)}\|z\|_{\boldsymbol{H}_{D}^{1+\kappa}(\Omega)} \\
& \leq C h^{2 \kappa}\left\|\boldsymbol{u}_{\tau, m}\right\|_{\boldsymbol{H}_{D}^{1+\kappa}(\Omega)}\left\|\boldsymbol{u}_{\tau, m}-\tilde{\boldsymbol{u}}_{\tau h, m}\right\|_{L^{2}(\Omega)} .
\end{aligned}
$$

Finally, with the stability estimate (3.9) for $\left\|\boldsymbol{u}_{\tau, m}\right\|_{\boldsymbol{H}_{D}^{1+\kappa}(\Omega)}$ we have the following result:

Lemma 4.9. Let Assumption 2.1 be fulfilled. Let $\boldsymbol{u}_{\tau, m}=\mathcal{U}\left(P_{\tau} l_{I_{m}}, \Phi\left(P_{\tau} l_{I_{m}}, d_{\tau, m}\right)\right) \in \boldsymbol{W}_{D}^{1, p}(\Omega)$ be the solution of (4.4) and let $\boldsymbol{u}_{\tau h, m}=\mathcal{U}_{h}\left(P_{\tau} l_{I_{I m}}, \Phi_{h}\left(P_{\tau} l_{\left.\right|_{I_{m}}}, d_{\tau h, m}\right)\right) \in \boldsymbol{U}_{h}^{1}$ be the solution of (4.5) for a given load $l \in$ $H^{1}\left(I ; \boldsymbol{L}^{2}(\Omega)\right) \cap \mathcal{B}_{\rho}^{1,2}$ and given $d_{\tau, m} \in L^{2}(\Omega), d_{\tau h, m} \in X_{h}^{1}$. Then, we have the error estimate

$$
\left\|\boldsymbol{u}_{\tau, m}-\boldsymbol{u}_{\tau h, m}\right\|_{L^{2}(\Omega)} \leq C\left(h^{\min \left\{2 \kappa, \theta+1-\frac{2}{p}\right\}}+L_{\Phi}\left\|d_{\tau, m}-d_{\tau h, m}\right\|_{L^{2}(\Omega)}\right)
$$

with a constant $C>0$ independent of $\tau, h$ and the subinterval $I_{m}$ for each $m=1, \ldots, M$.

$L^{2}\left(I ; L^{2}(\Omega)\right)$-error estimates for $d_{\tau}-d_{\tau h}$

Error estimates for $\boldsymbol{u}_{\tau, m}-\boldsymbol{u}_{\tau h, m}$ and $\varphi_{\tau, m}-\varphi_{\tau h, m}$ thus only depend on an error estimate for $d_{\tau, m}-d_{\tau h, m}$ in $L^{2}(\Omega)$. We are going to establish such an error estimate in the following. Note, that the approach is highly linked to [19] as the problem discussed in the next paragraph and the problem discussed in Section 3.2 of [19], only differ in the PDE underlying the solution operator $\Phi$. We thus only state important results.

We start with the definition of a reduced bilinear form $\mathfrak{b}$ :

Definition 4.10. The reduced bilinear form $\mathfrak{b}: X_{\tau}^{0} \times X_{\tau}^{0} \rightarrow \mathbb{R}$ is given as

$$
\mathfrak{b}\left(d_{\tau}, \lambda\right):=\sum_{m=2}^{M}\left(\left[d_{\tau}\right]_{m-1}, \lambda_{m-1}^{+}\right)+\left(d_{\tau, 0}^{+}, \lambda_{0}^{+}\right) .
$$


Then recall, that $d_{\tau} \in X_{\tau}^{0}$ is the solution of the semidiscrete problem

$$
\mathfrak{b}\left(d_{\tau}, \lambda\right)=\frac{1}{\delta}\left(\max \left(-\beta\left(d_{\tau}-\varphi_{\tau}\right)-r\right), \lambda\right)_{I \times \Omega}+\left(d_{0}, \lambda_{0}^{+}\right)
$$

with $\varphi_{\tau}=\Phi\left(P_{\tau} l, d_{\tau}\right)$ for all $\lambda \in X_{\tau}^{0}$ while $d_{\tau h} \in X_{\tau h}^{0,1}$ solves

$$
\mathfrak{b}\left(d_{\tau h}, \lambda\right)=\frac{1}{\delta}\left(\max \left(-\beta\left(d_{\tau h}-\varphi_{\tau h}\right)-r\right), \lambda\right)_{I \times \Omega}+\left(d_{0}, \lambda_{0}^{+}\right)
$$

with $\varphi_{\tau h}=\Phi_{h}\left(P_{\tau} l, d_{\tau h}\right)$ for all $\lambda \in X_{\tau h}^{0,1}$.

As in the previous paragraphs, we split the error as $d_{\tau}-d_{\tau h}=d_{\tau}-\tilde{d}_{\tau h}+\tilde{d}_{\tau h}-d_{\tau h}$ where the auxiliary solution $\tilde{d}_{\tau h} \in X_{\tau h}^{0,1}$ is defined as the solution of

$$
\mathfrak{b}\left(\tilde{d}_{\tau h}, \lambda\right)=\frac{1}{\delta}\left(\max \left(-\beta\left(\tilde{d}_{\tau h}-\varphi_{\tau}\right)-r\right), \lambda\right)_{I \times \Omega}+\left(d_{0}, \lambda_{0}^{+}\right)
$$

for all $\lambda \in X_{\tau h}^{0,1}$. Unique solvability of (4.26) and thus also of (4.27) can be proven as in the semidiscrete case with Banach's fixed point theorem and again requires that $\tau_{m}$ is sufficiently small. An estimate for $d_{\tau}-\tilde{d}_{\tau h}$ has already been established in Theorem 3.15 of [19]. Therefore, we only state the result:

Lemma 4.11. Let $d_{\tau} \in X_{\tau}^{0}$ be the solution of (4.25) and let $\tilde{d}_{\tau h} \in X_{\tau h}^{0,1}$ be the solution of (4.27) for a given right-hand side $l \in H^{1}\left(I ; \boldsymbol{L}^{2}(\Omega)\right) \cap \mathcal{B}_{\rho}^{1,2}$ and a given initial value $d_{0} \in H^{s}(\Omega), s=\min \left\{1+\theta, \frac{3}{2}-\epsilon\right\}$. Then, we have the error estimate

$$
\left\|d_{\tau}-\tilde{d}_{\tau h}\right\|_{I \times \Omega} \leq C h^{s}\left\|d_{\tau}\right\|_{L^{2}\left(I ; H^{s}(\Omega)\right)}
$$

with a constant $C>0$ independent of $\tau$ and $h$.

The required regularity for $d_{\tau}$ as well as uniform boundedness in $L^{2}\left(I ; H^{s}(\Omega)\right)$ is proven in Theorem 3.1 and Lemma 3.3. An estimate for the second term is given in [19] as well, see Lemma 3.9. The required preliminary error estimate for $\varphi_{\tau, m}-\varphi_{\tau h, m}$ in $L^{2}(\Omega)$ is given by Lemma 4.8 .

Lemma 4.12. Let $\tilde{d}_{\tau h} \in X_{\tau h}^{0,1}$ be the solution of (4.27) and let $d_{\tau h} \in X_{\tau h}^{0,1}$ be the solution of (4.26) for a given right-hand side $l \in H^{1}\left(I ; \boldsymbol{L}^{2}(\Omega)\right) \cap \mathcal{B}_{\rho}^{1,2}$ and an initial state $d_{0} \in L^{2}(\Omega)$. Then, we have the error estimate

$$
\left\|\tilde{d}_{\tau h}-d_{\tau h}\right\|_{L^{\infty}\left(I ; L^{2}(\Omega)\right)} \leq C\left\{h^{2 \theta}+\left\|d_{\tau}-\tilde{d}_{\tau h}\right\|_{L^{1}\left(I ; L^{2}(\Omega)\right)}\right\}
$$

with a constant $C>0$ independent of $\tau$ and $h$.

The results so far can now be combined to

Theorem 4.13. Let Assumption 2.1 be fulfilled. Let $\left(\boldsymbol{u}_{\tau}, \varphi_{\tau}, d_{\tau}\right) \in \boldsymbol{U}_{\tau}^{0}(p) \times V_{\tau}^{0} \times X_{\tau}^{0}$ be the solution of (3.1)-(3.3) and let $\left(\boldsymbol{u}_{\tau h}, \varphi_{\tau h}, d_{\tau h}\right) \in \boldsymbol{U}_{\tau h}^{0,1} \times X_{\tau h}^{0,1} \times X_{\tau h}^{0,1}$ be the solution of $(4.1)-(4.3)$ for a given load $l \in H^{1}\left(I ; \boldsymbol{L}^{2}(\Omega)\right) \cap \mathcal{B}_{\rho}^{1,2}$ and an initial state $d_{0} \in H^{s}(\Omega), s=\min \left\{1+\theta, \frac{3}{2}-\varepsilon\right\}$. Then, we have the error estimates

$$
\begin{aligned}
\left\|d_{\tau}-d_{\tau h}\right\|_{I \times \Omega} & \leq C h^{\min \left\{\frac{3}{2}-\epsilon, 2 \theta\right\}}, \\
\left\|\boldsymbol{u}_{\tau}-\boldsymbol{u}_{\tau h}\right\|_{L^{2}\left(I ; \boldsymbol{W}_{D}^{1,2}(\Omega)\right)} & \leq C h^{\kappa} \\
\left\|\varphi_{\tau}-\varphi_{\tau h}\right\|_{L^{2}\left(I ; H^{1}(\Omega)\right)} & \leq C h^{\theta} \\
\left\|\boldsymbol{u}_{\tau}-\boldsymbol{u}_{\tau h}\right\|_{I \times \Omega} & \leq C h^{\min \left\{2 \kappa, \frac{3}{2}-\epsilon\right\}}, \\
\left\|\varphi_{\tau}-\varphi_{\tau h}\right\|_{I \times \Omega} & \leq C h^{\min \left\{2 \theta, \frac{3}{2}-\epsilon\right\}},
\end{aligned}
$$

with a constant $C>0$ independent of $\tau$ and $h$. 
Proof. At first, the combination of Lemmas 4.11 and 4.12 yields

$$
\left\|d_{\tau}-d_{\tau h}\right\|_{I \times \Omega} \leq C h^{\min \left\{1+\theta, \frac{3}{2}-\epsilon, 2 \theta\right\}} .
$$

Since $1+\theta \geq 2 \theta$, we arrive at (4.30). Next, we obtain the third estimate by building $L^{2}(I ; \cdot)$-norms on both sides of (4.15). This gives us

$$
\left\|\varphi_{\tau}-\varphi_{\tau h}\right\|_{L^{2}\left(I ; H^{1}(\Omega)\right)} \leq C h^{\min \left\{\theta, \frac{3}{2}-\epsilon, 2 \theta\right\}} .
$$

Since $\theta \leq 2 \theta$ and $\theta \leq 1<\frac{3}{2}-\epsilon$, the minimum is attained in $\theta$. Then, building $L^{2}(I ; \cdot)$-norms on both sides of (4.8) together with the estimate derived in (4.20) for $\varphi_{\tau, m}-\varphi_{\tau h, m}$ in $L^{\frac{2 p}{p-2}}(\Omega)$ yields an error estimate for $\boldsymbol{u}_{\tau}-\boldsymbol{u}_{\tau h}$ in $L^{2}\left(I ; \boldsymbol{W}_{D}^{1,2}(\Omega)\right)$. We obtain

$$
\left\|\boldsymbol{u}_{\tau}-\boldsymbol{u}_{\tau h}\right\|_{L^{2}\left(I ; \boldsymbol{W}_{D}^{1,2}(\Omega)\right)} \leq C h^{\min \left\{\kappa, \theta+1-\frac{2}{p}, \frac{3}{2}-\epsilon, 2 \theta\right\}} .
$$

Again, we note that $\theta+1-\frac{2}{p} \leq 2 \theta$ in both cases $\theta=1$ and $\theta=2-\frac{4}{p}$ which is why we can omit $2 \theta$. Moreover, we have $\kappa \leq 1$ which means that we can eliminate $\frac{3}{2}-\epsilon$ as well. Finally, due the restriction (2.20), we have $\kappa \leq 1-\frac{2}{p}$. Since $\theta>0$ we arrive at the second estimate. Next, we build norms on both sides of (4.23) and obtain

$$
\left\|\boldsymbol{u}_{\tau}-\boldsymbol{u}_{\tau h}\right\|_{I \times \Omega} \leq C h^{\min \left\{2 \kappa, \theta+1-\frac{2}{p}, \frac{3}{2}-\epsilon\right\}} .
$$

This time, we consider different cases with respect to $\theta$. If $\theta=2-\frac{4}{p}$, then due to $1-\frac{2}{p} \geq \kappa$ we obtain $\theta \geq 2 \kappa$ and thus $\theta+1-\frac{2}{p} \geq 3 \kappa$. If $\theta=1$, which is equivalent to $p \geq 4$, we obtain $\theta+1-\frac{2}{p}=2-\frac{2}{p} \geq 2-\frac{2}{4}=\frac{3}{2}>\frac{3}{2}-\epsilon$. Thus, the minimum is never attained at $\theta+1-\frac{2}{p}$. This gives us the fourth estimate. Building corresponding norms on both sides of (4.19) finally yields the last estimate.

In a nutshell, Theorem 4.13 tells us, that the $L^{2}\left(I ; H^{1}(\Omega)\right)$-errors with respect to $\boldsymbol{u}$ and $\varphi$ converge of order $\kappa$ and $\theta$ as one would expect due to the regularity of the solutions. This rate is doubled if we consider $L^{2}\left(I ; L^{2}(\Omega)\right)$-norms unless the regularity of $d_{\tau}$ comes into play and limits the order of convergence. The other way round, the error for $d_{\tau}-d_{\tau h}$ in $L^{2}\left(I ; L^{2}(\Omega)\right)$ converges with order $\frac{3}{2}-\epsilon$ unless the order of convergence for $\varphi_{\tau}-\varphi_{\tau h}$ in $L^{2}\left(I ; L^{2}(\Omega)\right)$ is worse.

A combination of Theorems 4.13 and 3.8 together with triangle inequality then yields overall error estimates:

Corollary 4.14. Let Assumption 2.1 be fulfilled. For a given load $l \in H^{1}\left(I ; \boldsymbol{L}^{2}(\Omega)\right) \cap \mathcal{B}_{\rho}^{1,2}$ and an initial state $d_{0} \in H^{s}(\Omega), s=\min \left\{1+\theta, \frac{3}{2}-\varepsilon\right\}$, let $(\boldsymbol{u}, \varphi, d) \in L^{\infty}\left(I ; \boldsymbol{W}_{D}^{1,2}(\Omega)\right) \times L^{\infty}\left(I ; H^{1}(\Omega)\right) \times W^{1, \infty}\left(I ; L^{2}(\Omega)\right)$ be the solution of the continuous damage model and let $\left(\boldsymbol{u}_{\tau h}, \varphi_{\tau h}, d_{\tau h}\right) \in \boldsymbol{U}_{\tau h}^{0,1} \times X_{\tau h}^{0,1} \times X_{\tau h}^{0,1}$ be the solution of (4.1)-(4.3). Then, we have the error estimates

$$
\begin{gathered}
\left\|d-d_{\tau h}\right\|_{I \times \Omega} \leq C\left(\tau+h^{\min \left\{\frac{3}{2}-\epsilon, 2 \theta\right\}}\right), \\
\left\|\boldsymbol{u}-\boldsymbol{u}_{\tau h}\right\|_{I \times \Omega} \leq C\left(\tau+h^{\min \left\{2 \kappa, \frac{3}{2}-\epsilon\right\}}\right), \\
\left\|\varphi-\varphi_{\tau h}\right\|_{I \times \Omega} \leq C\left(\tau+h^{\min \left\{2 \theta, \frac{3}{2}-\epsilon\right\}}\right),
\end{gathered}
$$

with a constant $C>0$ independent of $\tau$ and $h$.

\section{Numerical EXAMPLE}

In this last section, we have a look at a numerical example for the simulation of our damage model. We apply a fixed point argument to the reduced ODE to solve the model equations. In each time step, the solutions of the PDE system $\boldsymbol{u}$ and $\varphi$ are computed with a Newton solver. 

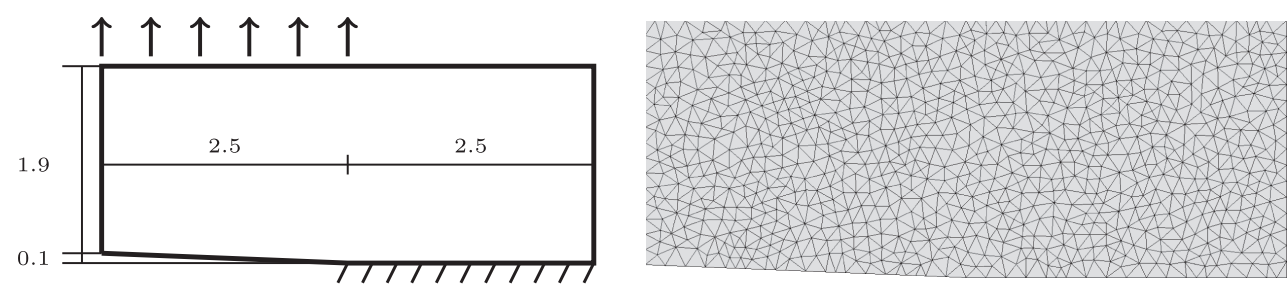

FiguRE 1. Left: geometry of the 2nd example, Right: mesh with 911 nodes .

We work with isotropic materials and assume that our object is completely sound at the beginning of our observation, that is $d_{0}=0$.

We consider an example with a non-fictitious material. It is based on a sharp notch problem presented in [21].

The geometry of the domain is illustrated in Figure 1 with all lengths given in $\mathrm{cm}$. Dirichlet boundary conditions are defined on $\Gamma_{D}=\{(x, y) \in \Omega: y=0\}$. The load (given in MPa)

$$
l(t, x, y)= \begin{cases}0.5(0,1)^{\top}, & \text { if }(x, y) \in[0,2.5] \times\{2\} \\ (0,0)^{\top}, & \text { else, }\end{cases}
$$

is applied on the left part of the top boundary. We set $\alpha=1, \beta=10, \delta=0.1$ and choose $r=0.1$. The Lamé parameters are chosen as $\mu_{L}=\frac{E}{2(1+\nu)}$ and $\lambda_{L}=\frac{E \nu}{(1-2 \nu)(1+\nu)}$ for Young's modulus $E=2000 \mathrm{MPa}$ and Poisson's ratio $\nu=0.33$. The magnitude of the load is chosen so that, at the beginning of the simulation, we have a small active set $\Omega_{+}^{0}=\left\{x \in \Omega: \varphi(0) \geq \frac{r}{\beta}\right\}$. If the active set was empty at the beginning of the observation, damage would not occur since the load is chosen independent of time. Note, that our findings cannot be compared to the results from [21] since the authors chose a time-dependent displacement and different material parameters.

The exact solution is unknown. For each experiment, we compute a reference solution $(\hat{\boldsymbol{u}}, \hat{\varphi}, \hat{d})$ on a fine temporal or spatial grid. In both cases, the temporal discretization parameter has to be chosen small since, otherwise, the fixed point argument fails. The reason for this is the choice of $\beta$. We choose $\beta$ larger than 1 because numerical tests show that the Newton solver cannot compute a solution of the PDE system otherwise. Therefore, this example also illustrates the theoretical results of [32] about the unique solvability of the PDE system.

Figure 2 depicts the displacement and the development of both damage functions. The object is rotated by $180^{\circ}$ for the visualization of the damage. The pictures of the nonlocal damage variable $\varphi$ in the middle row show that there are three areas where damage might occur: the singular corner in the middle of the bottom boundary, the bottom right corner and the area in the middle of the top boundary. But $\varphi$ exceeds the threshold $\frac{r}{\beta}$ only at the singular corner. Consequently, damage occurs in this area only. The bottom row depicts the development of the local damage $d$.

For the visualization of the behavior of the temporal error, we compute the reference solution $(\hat{\boldsymbol{u}}, \hat{\varphi}, \hat{d})$ for $N_{h}=3605$ and $M=2^{12}$. For the visualization of the behavior of the spatial error, the reference solution is computed for $N_{h}=56684$ and $M=2^{10}$. We denote errors by $e_{\tau h}^{\boldsymbol{u}}:=\hat{\boldsymbol{u}}-\boldsymbol{u}_{\tau h}$.

Table 1 depicts the development of the temporal discretization errors. We observe first order convergence for all three functions. Tables 2 and 3 depict the development of the spatial $L^{2}\left(I ; L^{2}(\Omega)\right)$ - and $L^{2}\left(I ; H^{1}(\Omega)\right)$-errors. At first we note, that the results are not perfect. There are some values for the experimental order of convergence (marked in italic) that are outliers. They were omitted from the calculation of the mean. Nonetheless, the results underline our theoretical findings of Theorem 4.13. We observe a rate of convergence in $L^{2}\left(I ; H^{1}(\Omega)\right)$ that is less than 1 for the errors in $\boldsymbol{u}$ and $\varphi$. This rate is approximately doubled when we consider $L^{2}\left(I ; L^{2}(\Omega)\right.$ )-errors. All $L^{2}\left(I ; L^{2}(\Omega)\right)$-errors converge with a rate that is less than $\frac{3}{2}$. And finally, the $L^{2}\left(I ; L^{2}(\Omega)\right)$-errors for $\varphi$ and $d$ converge with the same rate (Fig. 3-5). 


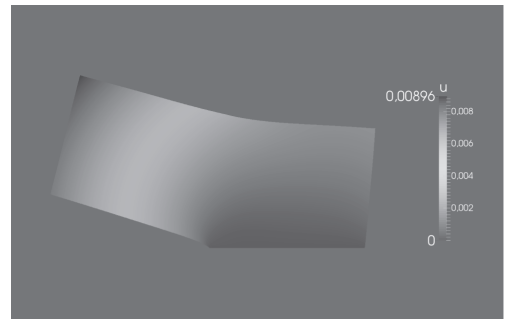

(a)

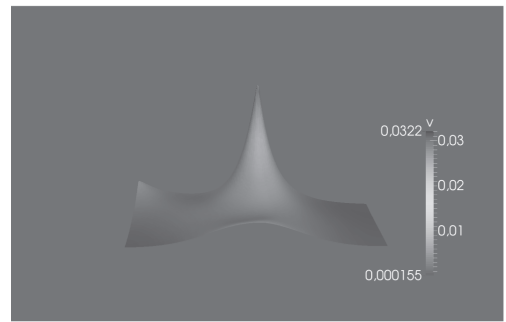

(d)

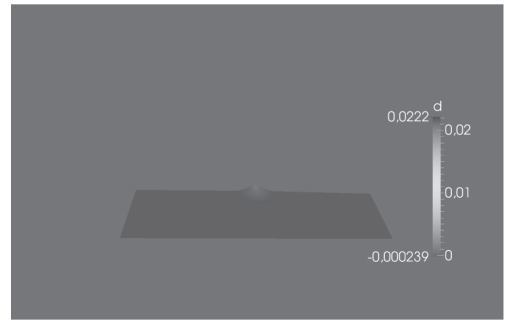

(g)

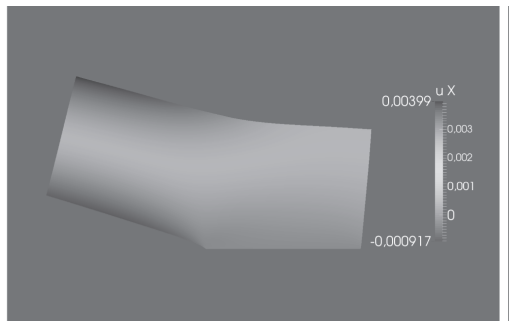

(b)

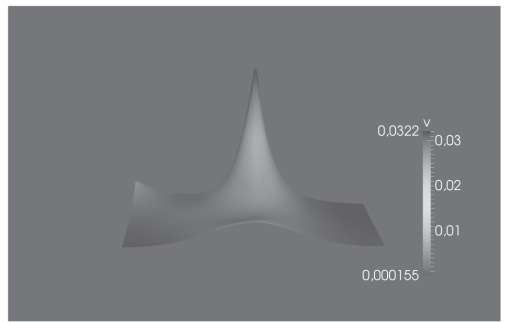

(e)

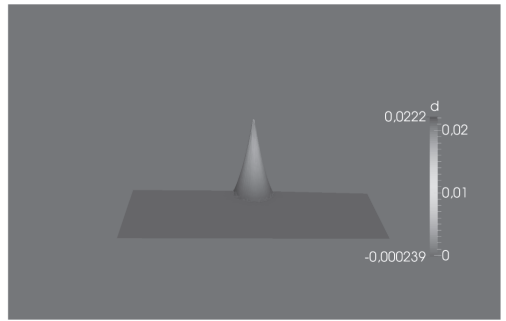

(h)

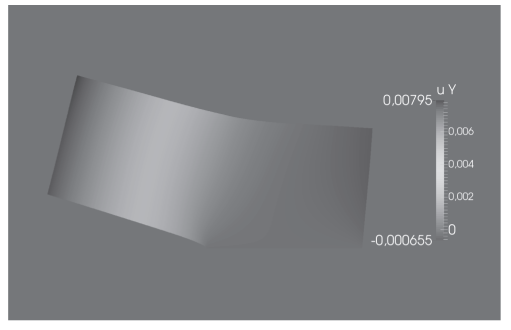

(c)

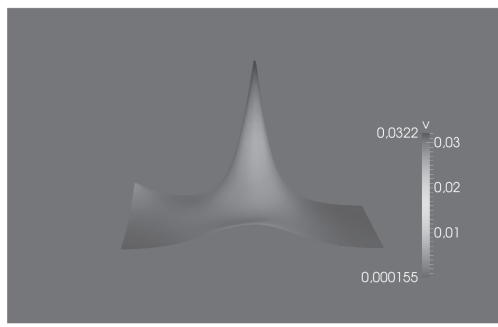

(f)

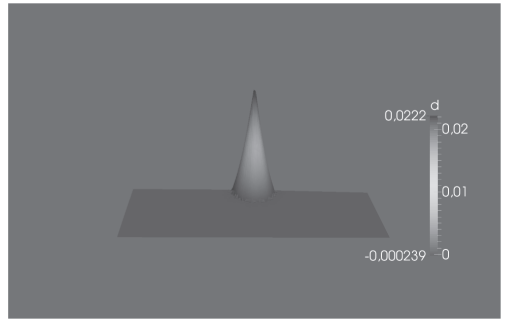

(i)

Figure 2. The computed displacement $\boldsymbol{u}$ (top), nonlocal damage $\varphi$ (middle) and local damage $d$ (bottom) for $N_{h}=3605$ nodes and $M=1024$. The object is rotated by $180^{\circ}$ in the middle and bottom row. Development of displacement is not visible, damage develops at the very beginning of the observation period (approximately 200 of 1024 iterations) and afterwards does not change visibly. All three functions are shown 100 times enlarged (a) $\left|\left(u_{x}, u_{y}\right)\right|$ (b) $u_{x}$ (c) $u_{y}$ (d) $T=9.76 e-05$ (e) $T=1.95 e-02$ (f) $T=1.95 e-01$ (g) $T=9.76 e-05$ (h) $T=1.95 e-02$ (i) $T=1.95 e-01$.

TABLE 1. 2nd example: $L^{2}\left(I ; L^{2}(\Omega)\right)$-errors for the states for a temporal refinement.

\begin{tabular}{lllllll}
\hline \hline$N_{h}$ & \multicolumn{7}{c}{3605} \\
\hline$\tau$ & $\left\|e_{\tau h}^{\boldsymbol{u}}\right\|_{I \times \Omega}$ & EOC & $\left\|e_{\tau h}^{\varphi}\right\|_{I \times \Omega}$ & EOC & $\left\|e_{\tau h}^{d}\right\|_{I \times \Omega}$ & EOC \\
\hline $2^{-7}$ & $2.38 \mathrm{e}-07$ & - & $3.81 \mathrm{e}-05$ & - & $8.11 \mathrm{e}-05$ & - \\
$2^{-8}$ & $1.27 \mathrm{e}-07$ & 0.90 & $2.03 \mathrm{e}-05$ & 0.90 & $4.39 \mathrm{e}-05$ & 0.88 \\
$2^{-8}$ & $8.38 \mathrm{e}-08$ & 0.60 & $1.32 \mathrm{e}-05$ & 0.61 & $2.11 \mathrm{e}-05$ & 1.05 \\
$2^{-10}$ & $4.48 \mathrm{e}-08$ & 0.90 & $7.10 \mathrm{e}-06$ & 0.90 & $9.29 \mathrm{e}-06$ & 1.18 \\
$2^{-11}$ & $2.18 \mathrm{e}-08$ & 1.04 & $3.41 \mathrm{e}-06$ & 1.05 & $3.80 \mathrm{e}-06$ & 1.28 \\
\hline
\end{tabular}


TABLE 2. 2nd example: $L^{2}\left(I ; L^{2}(\Omega)\right)$-errors for the states for a spatial refinement.

\begin{tabular}{llllllll}
\hline \hline \multicolumn{7}{c}{$\tau$} & \multicolumn{5}{c}{$2^{-10}$} \\
\hline$N_{h}$ & $h$ & $\left\|e_{\tau h}^{u}\right\|_{I \times \Omega}$ & EOC & $\left\|e_{\tau h}^{\varphi}\right\|_{I \times \Omega}$ & EOC & $\left\|e_{\tau h}^{d}\right\|_{I \times \Omega}$ & EOC \\
\hline 18 & $1.05 \mathrm{e}-00$ & $7.88 \mathrm{e}-04$ & - & $4.98 \mathrm{e}-03$ & - & $2.57 \mathrm{e}-03$ & - \\
64 & $5.35 \mathrm{e}-01$ & $7.40 \mathrm{e}-04$ & 0.09 & $2.26 \mathrm{e}-03$ & 1.17 & $2.00 \mathrm{e}-03$ & 0.37 \\
236 & $2.68 \mathrm{e}-01$ & $2.47 \mathrm{e}-04$ & 1.58 & $2.04 \mathrm{e}-03$ & 0.14 & $1.53 \mathrm{e}-03$ & 0.38 \\
911 & $1.34 \mathrm{e}-01$ & $9.10 \mathrm{e}-05$ & 1.44 & $9.54 \mathrm{e}-04$ & 1.10 & $7.46 \mathrm{e}-04$ & 1.04 \\
3605 & $6.73 \mathrm{e}-02$ & $4.89 \mathrm{e}-05$ & 0.89 & $4.45 \mathrm{e}-04$ & 1.10 & $3.50 \mathrm{e}-04$ & 1.09 \\
14203 & $3.36 \mathrm{e}-02$ & $8.63 \mathrm{e}-05$ & -0.81 & $1.78 \mathrm{e}-04$ & 1.31 & $1.41 \mathrm{e}-04$ & 1.31 \\
\hline \multicolumn{7}{c}{} \\
$\varnothing$ & & 1.30 & & 1.16 & & 1.14 \\
\hline
\end{tabular}

TABLE 3. 2nd example: $L^{2}\left(I ; H^{1}(\Omega)\right)$-errors for the states for a spatial refinement.

\begin{tabular}{llllll}
\hline \hline \multicolumn{5}{c}{$\tau$} & \multicolumn{5}{c}{$2^{-10}$} \\
\hline$N_{h}$ & $h$ & $\left\|e_{\tau h}^{\boldsymbol{u}}\right\|_{L^{2}\left(I ; H^{1}(\Omega)\right)}$ & $\mathrm{EOC}$ & $\left\|e_{\tau h}^{\varphi}\right\|_{L^{2}\left(I ; H^{1}(\Omega)\right)}$ & $\mathrm{EOC}$ \\
\hline 18 & $1.05 \mathrm{e}-00$ & $1.75 \mathrm{e}-03$ & - & $2.07 \mathrm{e}-01$ & - \\
64 & $5.35 \mathrm{e}-01$ & $1.23 \mathrm{e}-03$ & 0.51 & $1.48 \mathrm{e}-01$ & 0.49 \\
236 & $2.68 \mathrm{e}-01$ & $7.40 \mathrm{e}-04$ & 0.74 & $1.06 \mathrm{e}-01$ & 0.47 \\
911 & $1.34 \mathrm{e}-01$ & $4.69 \mathrm{e}-04$ & 0.65 & $6.63 \mathrm{e}-02$ & 0.69 \\
3605 & $6.73 \mathrm{e}-02$ & $3.09 \mathrm{e}-04$ & 0.60 & $3.69 \mathrm{e}-02$ & 0.84 \\
14203 & $3.36 \mathrm{e}-02$ & $2.39 \mathrm{e}-04$ & 0.36 & $2.04 \mathrm{e}-02$ & 0.85 \\
\hline \multicolumn{5}{c}{0.57} \\
\hline
\end{tabular}

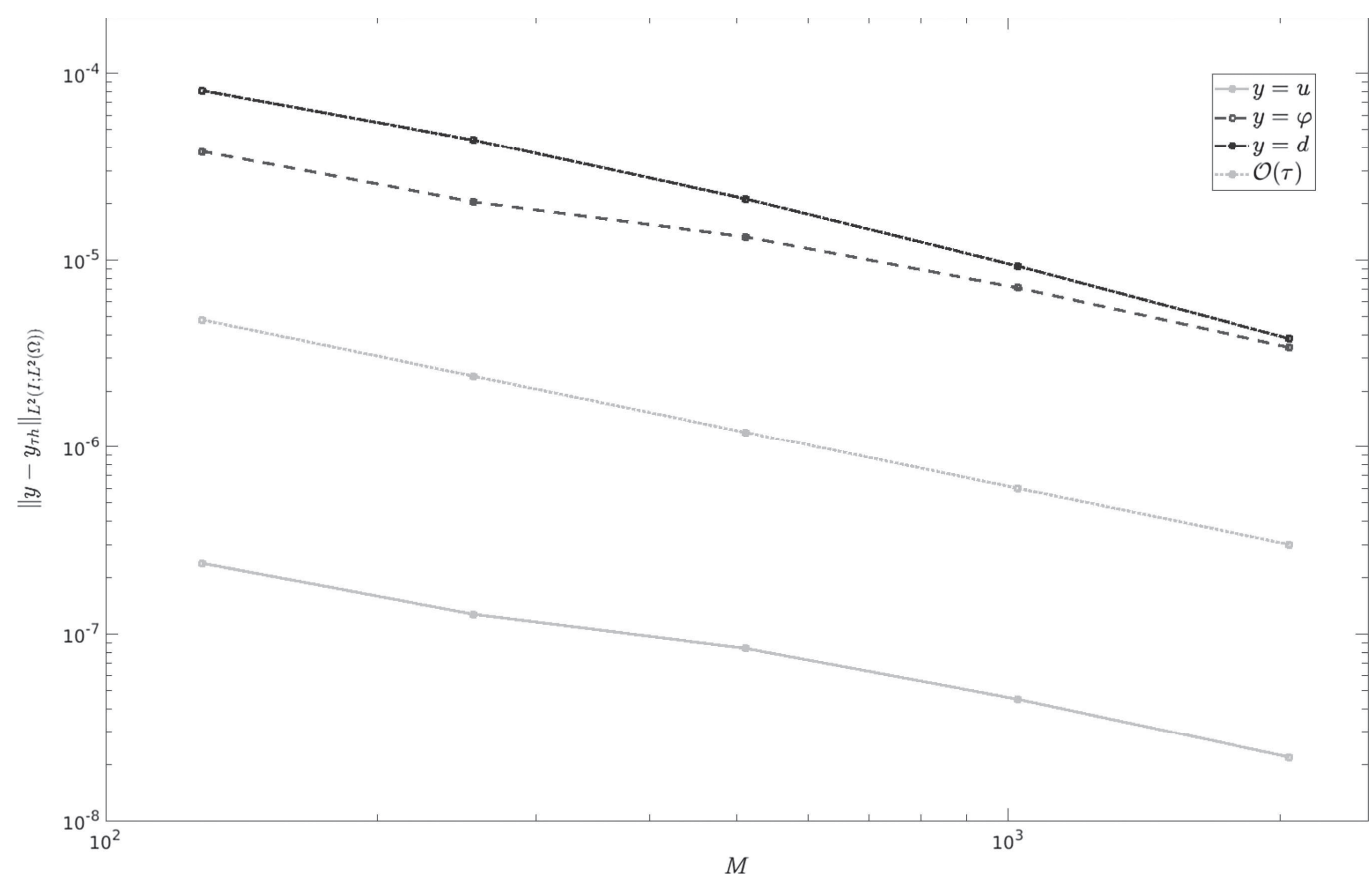

Figure 3. 2nd example: Errors for the states for a temporal refinement. 

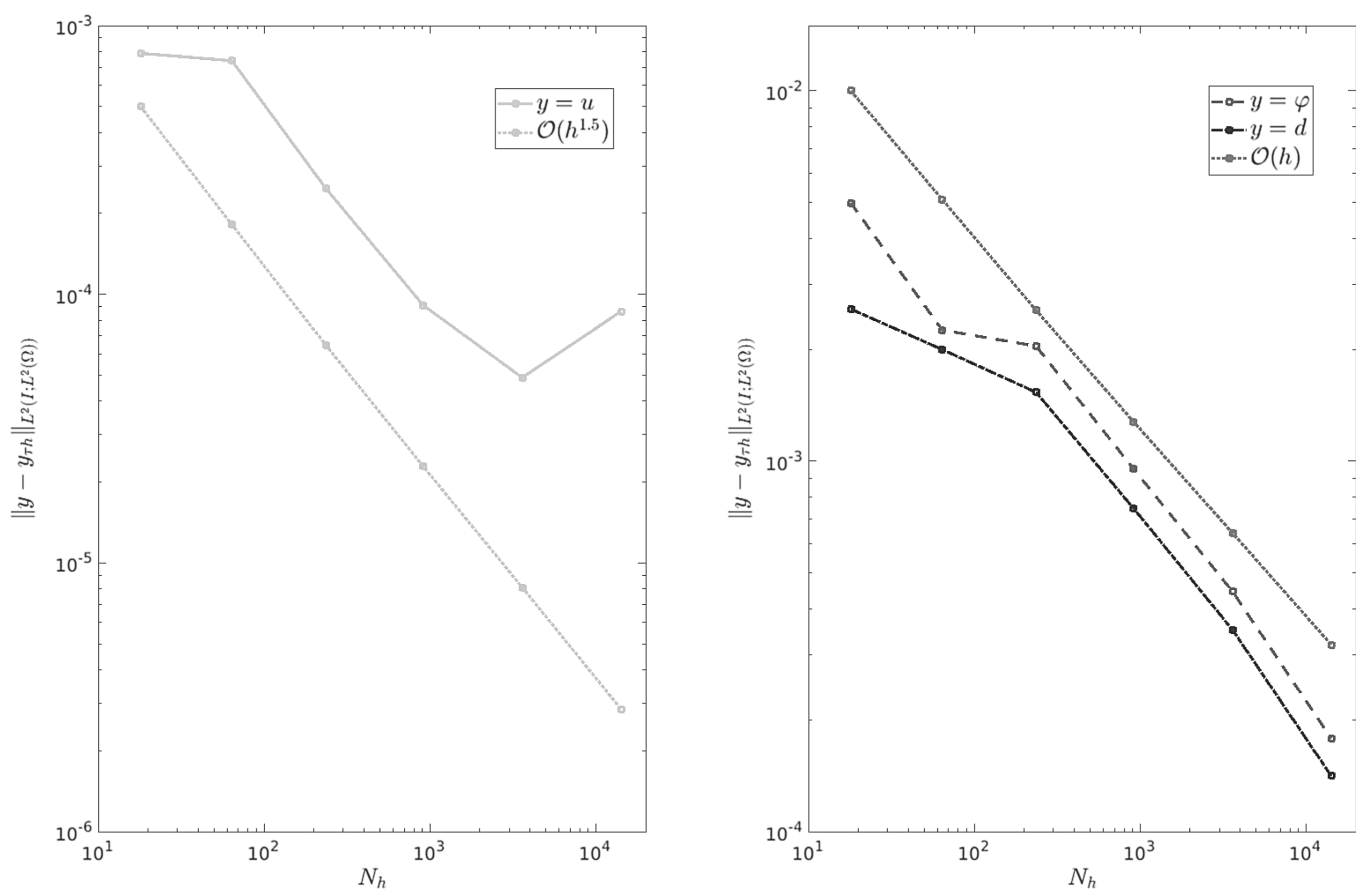

FiguRE 4. 2nd example: $L^{2}\left(I ; L^{2}(\Omega)\right)$-errors for the states for a spatial refinement.

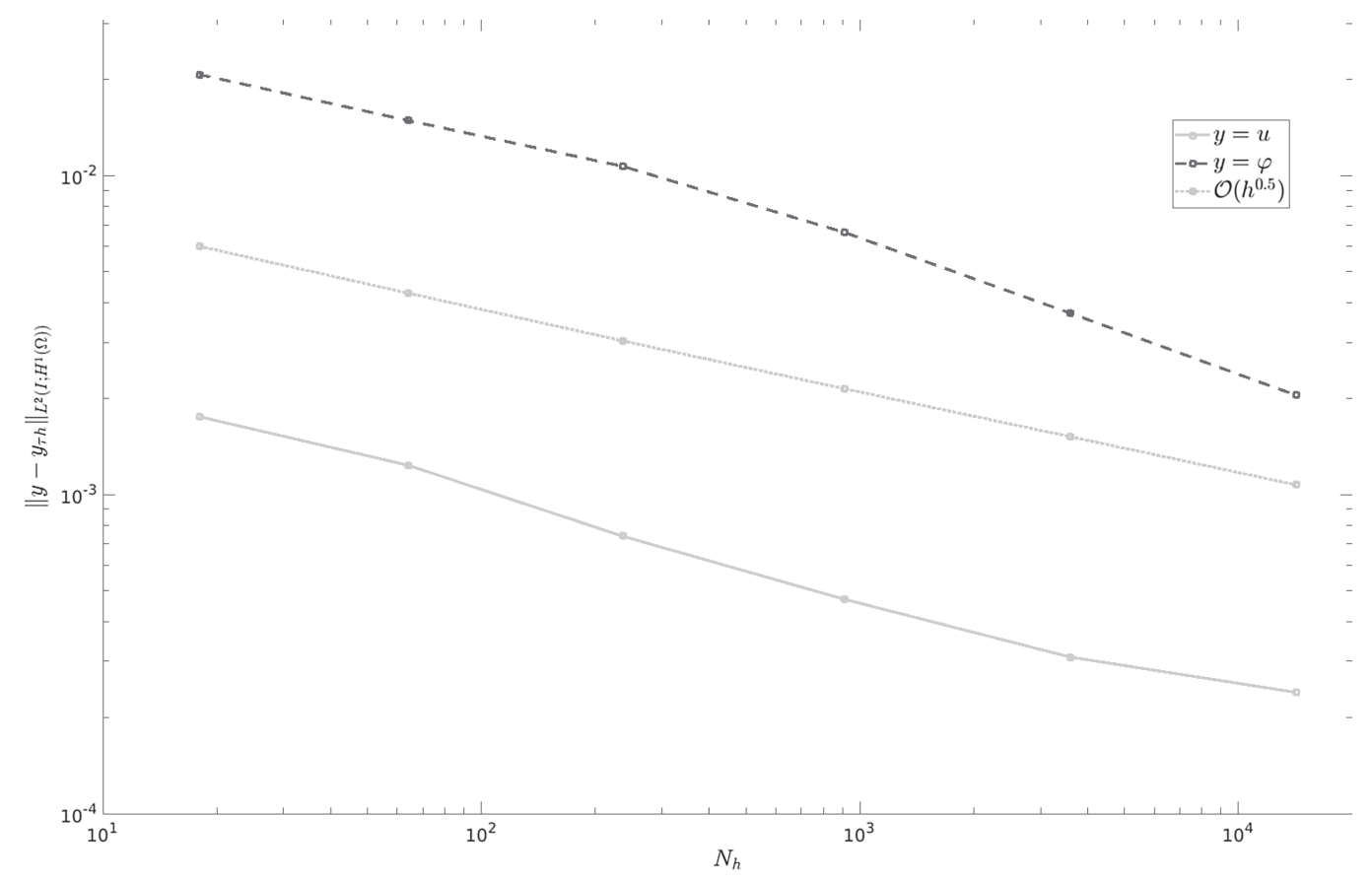

Figure 5. 2nd example: $L^{2}\left(I ; H^{1}(\Omega)\right)$-errors for the states for a spatial refinement. 
Acknowledgements. The author wants to thank the anonymous referees for the helpful comments which highly shortened the proof of Theorem 3.8 .

\section{REFERENCES}

[1] B. Bourdin, G.A. Francfort and J.J. Marigo, Numerical experiments in revisited brittle fracture. J. Mech. Phys. Solids 48 (2000) 797-826.

[2] S.C. Brenner and L.R. Scott, The Mathematical Theory of Finite Element Methods. Springer Verlag (2002).

[3] S. Burke, C. Ortner and E. Süli, An adaptive finite element approximation of a generalized Ambrosio-Tortorelli functional. Math. Models Methods Appl. Sci. 23 (2013) 1663-1697.

[4] G. Dal Maso, G.A. Francfort and R. Toader, Quasistatic crack growth in nonlinear elasticity. Arch. Rational Mech. Anal. 176 (2005) 165-225.

[5] B.J. Dimitrijevic and K. Hackl, A method for gradient enhancement of continuum damage models. Technische Mechanik, Ruhr-Universität Bochum 28 (2008) 43-52.

[6] B.J. Dimitrijevic and K. Hackl, A regularization framework for damage-plasticity models via gradient enhancement of the free energy. Int. J. Numer. Methods Biomed. Eng. 27 (2011) 1199-1210.

[7] M. Efendiev and A. Mielke, On the rate-independent limit of systems with dry friction and small viscosity. J. Convex Anal. 13 (2006) 151-167.

[8] E. Emmrich, Gewöhnliche und Operatordifferentialgleichungen. Berlin, Vieweg (2004).

[9] K. Eriksson and C. Johnson, Adaptive finite element methods for parabolic problems I: a linear model problem. SIAM J. Numer. Anal. 28 (1991) 43-77.

[10] K. Eriksson and C. Johnson, Adaptive finite element methods for parabolic problems II: optimal error estimates in $L^{\infty}\left(L^{2}\right)$ and $L^{\infty}\left(L^{\infty}\right)$. SIAM J. Numer. Anal. 32 (1995) 706-740.

[11] K. Eriksson and C. Johnson, Adaptive finite element methods for parabolic problems IV: Nonlinear problems. SIAM J. Numer. Anal. 32 (1995) 1729-1749.

[12] K. Eriksson, C. Johnson and V. Thomée, Time discretization of parabolic problems by the discontinuous Galerkin method. Rairo M.M.a.N 19 (1985) 611-643.

[13] D. Estep and S. Larsson, The discontinuous Galerkin method for semilinear parabolic problems. ESAIM: M2AN 27 (1993) $35-54$.

[14] G. Francfort and J.-J. Marigo, Revisiting brittle fracture as an energy minimization problem. J. Mech. Phys. Solids 46 (1998) $1319-1342$.

[15] G.A. Francfort and C.J. Larsen, Existence and convergence for quasi-static evolution in brittle fracture. Comm. Pure Appl. Math. 56 (2003) 1465-1500.

[16] A.A. Griffith, The phenomena of rupture and flow in solids. Philos. Trans. R. Soc. Lond. (1921)

[17] K. Gröger, A $W^{1, p}$-estimate for solutions to mixed boundary value problems for second order elliptic differential equations. Math. Ann. 283 (1989) 679-687.

[18] R. Haller-Dintelmann, H. Meinlschmidt and W. Wollner, Higher regularity for solutions to elliptic systems in divergence form subject to mixed boundary conditions. Annali di Matematica Pura ed Applicata (2018).

[19] M. Holtmannspötter and A. Rösch, A priori error estimates for the space-time finite element approximation of a non-smooth optimal control problem governed by a coupled semilinear PDE-ODE system. Preprint; arXiv:2004.05837 (2020).

[20] M. Holtmannspötter, A. Rösch and B. Vexler, A priori error estimates for the space-time finite element discretization of an optimal control problem governed by a coupled linear PDE-ODE system. Preprint; arXiv:2004.04448 (2020).

[21] P. Junker, S. Schwarz, D. Jantos and K. Hackl, A fast and robust numerical treatment of a gradient-enhanced model for brittle damage. Int. J. Multiscale Comput. Eng. 17 (2019) 151-180.

[22] D. Knees, R. Rossi and C. Zanini, A vanishing viscosity approach to a rate-independent damage model. Math. Models and Methods Appl. Sci., 23 (2013) 565-616.

[23] J. Makowski, K. Hackl and H. Stumpf, The fundamental role on nonlocal and local balance laws of material forces in finite elastoplasticity and damage mechanics. Int. J. Solids Struct. (2006).

[24] D. Meidner and B. Vexler, A priori error estimates for space-time finite element discretization of parabolic optimal control problems part I: Problems without control constraints. SIAM J. Control Optim. 47 (2008) 1150-1177.

[25] C. Meyer and L. Susu, Analysis of a viscous two-field gradient damage model, part I: Existence and uniqueness. ZAA 38 (2019) 249-286.

[26] C. Meyer and L. Susu, Analysis of a viscous two-field gradient damage model, part II: Penalization limit. ZAA 38 (2019) 439-474.

[27] M. Mohammadi and W. Wollner, A priori error estimates for a linearized fracture control problem. (2018) DOI: 10.1007/s11081-020-09574-z

[28] B. Nedjar, Elastoplastic-damage modelling including the gradient of damage: formulation and computational aspects. Int. J. Solids Struct. 38 (2001) 5421-5451. 
[29] I. Neitzel and B. Vexler, A priori error estimates for space-time finite element discretization of semilinear parabolic optimal control problems. Numerische Mathematik 120 (2011) 345-386.

[30] R. Peerlings, Enhanced damage modeling for fracture and fatigue. Ph.D. thesis, Technische Universiteit Eindhoven (1999).

[31] T. Runst and W. Sickel, Sobolev Spaces of Fractional Order, Nemytskij Operators, and Nonlinear Partial Differential Equations. de Gruyter (1996).

[32] L.M. Susu, Analysis and optimal control of a damage model with penalty. Ph.D. thesis, Technische Universität Dortmund (2017).

[33] L.M. Susu, Optimal control of a viscous two-field gradient damage model. GAMM-Mitteilungen 201840 (2018) $287-311$.

\section{Subscribe to Open (S20) A fair and sustainable open access model}

This journal is currently published in open access with no charge for authors under a Subscribe-to-Open model (S2O). Open access is the free, immediate, online availability of research articles combined with the rights to use these articles fully in the digital environment.

$\mathrm{S} 2 \mathrm{O}$ is one of the transformative models that aim to move subscription journals to open access. Every year, as long as the minimum amount of subscriptions necessary to sustain the publication of the journal is attained, the content for the year is published in open access.

\section{Ask your library to support open access by subscribing to this $\mathbf{S 2 O}$ journal.}

Please help to maintain this journal in open access! Encourage your library to subscribe or verify its subscription by contacting subscribers@edpsciences.org

We are thankful to our subscribers and sponsors for making it possible to publish the journal in open access, free of charge for authors. More information and list of sponsors: https://www.edpsciences.org/en/maths-s2o-programme 